\title{
Optimization of cw sodium laser guide star efficiency
}

\author{
R. Holzlöhner ${ }^{1}$, S. M. Rochester ${ }^{2}$, D. Bonaccini Calia ${ }^{1}$, D. Budker ${ }^{2}$, J. M. Higbie ${ }^{3}$, and W. Hackenberg ${ }^{1}$ \\ 1 Laser Systems Department, European Southern Observatory (ESO), Karl-Schwarzschild-Str. 2, 85748 Garching b. München, \\ Germany, http://www. eso.org/sci/facilities/develop/lgsf/ \\ e-mail: [rholzloe; dbonacci; whackenb] @eso.org \\ 2 University of California Berkeley, Department of Physics, Berkeley, CA 94720-7300, USA \\ e-mail: [simonr; budker] @berkeley.edu \\ 3 Bucknell University, Department of Physics, 701 Moore Avenue, Lewisburg, PA 17837, USA \\ e-mail: james.higbie@bucknell. edu
}

Received 12 August 2009 / Accepted 1 October 2009

ABSTRACT

\begin{abstract}
Context. Sodium laser guide stars (LGS) are about to enter a new range of laser powers. Previous theoretical and numerical methods are inadequate for accurate computations of the return flux, hence for the design of the next-generation LGS systems.

Aims. We numerically optimize the $\mathrm{cw}$ (continuous wave) laser format, in particular, the light polarization and spectrum.

Methods. Using Bloch equations, we simulate the mesospheric sodium atoms, including Doppler broadening, saturation, collisional relaxation, Larmor precession, and recoil, taking all 24 sodium hyperfine states into account and 100-300 velocity groups.

Results. LGS return flux is limited by "three evils": Larmor precession due to the geomagnetic field, atomic recoil due to radiation pressure, and transition saturation. We study their impact and show that the return flux can be boosted by repumping (simultaneous excitation of the sodium $\mathrm{D}_{2} \mathrm{a}$ and $\mathrm{D}_{2} \mathrm{~b}$ lines with 10-20\% of the laser power in the latter).

Conclusions. We strongly recommend the use of circularly polarized lasers and repumping. As a rule of thumb, the bandwidth of laser radiation in $\mathrm{MHz}$ (at each line) should approximately equal the launched laser power in Watts divided by six, assuming a diffraction-limited spot size.
\end{abstract}

Key words. instrumentation: adaptive optics - methods: numerical - atmospheric effects - telescopes

\section{Introduction}

Laser guide stars (LGS) are becoming essential in providing artificial beacons for adaptive optics (AO) in large telescopes (Hubin 2009). The generation of $8-10 \mathrm{~m}$ class telescopes, such as the Very Large Telescope (VLT) on Cerro Paranal, Chile, or the Keck telescopes on Mauna Kea, has been retrofitted with LGS and is still operated in many observing programs with natural guide stars or no $\mathrm{AO}$ at all. The upcoming $30+\mathrm{m}$ telescopes, such as the 42-m European Extremely Large Telescope (E-ELT) or the Thirty Meter Telescope (TMT), by contrast, are being designed from the start as adaptive telescopes and will require LGS in nearly all of their science operations. At the same time, sensing subaperture sizes of $20-50 \mathrm{~cm}$ and near-kHz AO frame rates, designed to provide high Strehl ratios in the infrared or even in the visible, require unprecedented LGS brightness and hence laser power.

Sodium (Na) LGS at $589 \mathrm{~nm}$ are most commonly used because of the large fluorescence cross section-abundance product of ca. $10^{-11} \mathrm{~cm}^{2} \times 4 \times 10^{13} \mathrm{~m}^{-2}$, a fluorescence wavelength in the visible, and the high altitude of the sodium layer around $80-100 \mathrm{~km}$ compared e.g. to Rayleigh LGS, (Happer 1994, Table 1), which allows one to sense a large fraction of the turbulent atmosphere column above the telescope with a small number of guide stars. Unfortunately, powerful diffractionlimited laser beams at $589 \mathrm{~nm}$ are quite expensive to produce because of the lack of solid-state materials that amplify $589 \mathrm{~nm}$ or $1178 \mathrm{~nm}$ light. Recently, ESO has demonstrated a frequencydoubled narrow-band Raman fiber laser emitting $25 \mathrm{~W}$ at $589 \mathrm{~nm}$ (Taylor 2009), and we expect this technology to significantly improve the experimental situation.
Careful dimensioning of the required laser power and optimization of the laser format (spectrum, polarization, spot size) are needed. Numerical simulations are necessary since the experimental situation has so far been unsatisfactory: the sodium layer and atmospheric parameters often fluctuate rapidly (Thomas 2008; Pfrommer 2009), few reliable powerful lasers at $589 \mathrm{~nm}$ have been available up to now, and LGS sky experiments lack commonly agreed on measurement standards. Sodium cells cannot easily simulate mesospheric conditions. This work provides optimization rules for the case of continuous wave (cw) lasers over a wide range of laser powers.

Sodium LGS take advantage of the $3^{2} S_{1 / 2}-3^{2} P_{3 / 2}$ dipole transition, known as the $\mathrm{D}_{2}$ line. The sodium ${ }^{2} S$ ground state consists of two hyperfine multiplets with 8 magnetic substates combined, separated by $1.772 \mathrm{GHz}$, splitting the $\mathrm{D}_{2}$ line into the $\mathrm{D}_{2} \mathrm{a}$ and $\mathrm{D}_{2} \mathrm{~b}$ transition groups, corresponding respectively to the $F=1$ and $F=2 \mathrm{Na}$ ground states, where $F$ is the total atomic angular momentum quantum number. The four ${ }^{2} P$ multiplets $(F=0 \ldots 3)$ are separated by only 16,34 , and $60 \mathrm{MHz}(16 \mathrm{mag}$ netic substates, see Ungar 1989). At mesospheric temperatures near $190 \mathrm{~K}$, the $\mathrm{D}_{2} \mathrm{a}$ and $\mathrm{D}_{2} \mathrm{~b}$ lines are Doppler broadened to about $1 \mathrm{GHz}$ each, giving rise to the characteristic double-hump absorption profile (see for instance Bradley 1992, Fig. 11, or Fig. 1 of this work). When the $\mathrm{D}_{2}$ a transition is excited by circularly polarized light at high irradiance (i.e. optical power density, unit $\mathrm{W} / \mathrm{m}^{2}$ ), a large fraction of atoms is pumped into the $(S, F=2, m= \pm 2)$ substate, and the atoms cycle on the transition to the $\left(P, F^{\prime}=3, m^{\prime}= \pm 3\right)$ substate, with $m$ the magnetic quantum number, so that sodium effectively becomes a two-level system. This situation is twofold desirable because of the large 
transition cross section and the directional return light peak towards the laser emitter and the telescope. Numerous studies of optical pumping have been conducted, e.g. by Happer (1972) and McClelland (1985), and the process is well understood.

The effective sodium return flux depends on the environmental conditions such as collisions with constituent gases, temperature, and the geomagnetic field. At higher irradiance, the atoms are driven away from thermal equilibrium, and it is necessary to take effects into account such as saturation, optical pumping, and the recoil caused by radiation pressure. Understanding the complicated interplay of these effects and obtaining quantitative values of the fluorescence efficiency requires numerical simulations. A commonly used method is the solution of the density matrix evolution (Bloch equation) of a multilevel atom.

Milonni \& Thode (1992) simplified the $\mathrm{D}_{2}$ scheme to a 2-level Bloch model which they solve in time domain. Bradley (1992) simulated the full 24-state density matrix, exciting the sodium by a train of short (nanosecond range) laser pulses like Milonni, using Runge-Kutta integration for one pulse period and exploiting the periodicity. Linear and circular light polarizations were treated. However, both works neglect the geomagnetic field. Morris (1994) studied frequency-modulated pulses over a wide range of pulse durations and linewidths up to $3 \mathrm{GHz}$, hence spanning the entire Doppler broadened $\mathrm{D}_{2}$ line, employing time domain integration, for both linearly and circularly polarized light. In the end of his paper, he briefly estimates the impact of the geomagnetic field, but he does not include Larmor precession terms into his Bloch equations.

To our knowledge, Milonni (1998, 1999) has published the most advanced and detailed Bloch-equation simulation of sodium LGS to date, later generalized by Telle (2006, 2008). In his 1998 article, Milonni treats the cases of laser pulses that are short, comparable, and long compared to the ${ }^{2} P$ lifetime of $\tau=16.24 \mathrm{~ns}$, using numerical solution methods similar to Bradley's. His 1999 publication deals with cw excitation only and introduces spin relaxation and Larmor precession into the Bloch equations for the first time. He shows that the Larmor terms due to the geomagnetic field tend to redistribute the magnetic sublevel populations and hence impede optical pumping. All of the works cited in this and the previous paragraph solve the Bloch equations separately for a number of different sodium velocity groups (100-400) and then perform a weighted average over the results, and all neglect recoil.

An alternative method of simulating atomic fluorescence is to use rate equations, either implemented as a set of differential equations, (Pique 2006), (Hillman 2008), or by employing Monte Carlo rate equation techniques ${ }^{1}$. In the latter, one follows a more intuitive approach and tracks the time evolution of a single atom, executing photon absorption and emission events according to steady-state cross sections and branching ratios using a random number generator, while accounting for elapsed physical time and recording the atomic state history. After a sufficient time span $\Delta t$ has been simulated, one divides the total number of spontaneously emitted photons by $\Delta t$ to obtain the fluorescent flux. The advantage of this method is that it is easy to understand, and complex physical events such as collisions with different kinds of particles with or without spin exchange can be modeled in a transparent way. Also, the transit of optically pumped atoms across the Doppler spectrum due to velocity-changing collisions and recoil can be easily modeled, hence different velocity

\footnotetext{
1 "Sodium Laser Guide Star Return Flux Study for The European Southern Observatory", contract study for ESO, E-TRE-KIB-297-0001, 26 August 2008 (available upon request).
}

groups can be properly coupled, including their atomic polarization exchange (using Bloch equations, such coupling requires the simultaneous solution of sets of $24^{2}$-dimensional equations per velocity group, which we describe in this work for the first time in application to sodium LGS, to our knowledge).

A serious disadvantage of rate equations is that they rely on steady-state atomic cross sections, limiting the scope to the simulation of events slow compared to the sodium transition lifetime, (Milonni 1992), and the combination of the excitationemission (Rabi) cycling with Larmor precession is hard to implement correctly. Furthermore, when compared to atomic Bloch equations, rate equations neglect the coherences (offdiagonal terms of the density matrix that describe transverse atomic polarization), which is problematic when modeling Larmor precession or linearly polarized light (the latter even for $B=0$ ). Finally, Monte Carlo rate-equations need considerable CPU time in order to converge.

There are also approaches that mix aspects of Bloch and rate-equation codes, such as BEACON, which has been adapted to model two-step sodium excitation for polychromatic LGS, (Bellanger 2004). BEACON neglects atomic collisions and recoil. Guillet de Chatellus (2008) reports that it requires on the order of $24 \mathrm{~h}$ per run on a $2.6 \mathrm{GHz}$ processor, and that the agreement with a pure rate-equation model can be good in certain cases.

Throughout this paper, we highlight what may be called the "three evils" of sodium LGS, ordered by decreasing importance

\section{Larmor precession,}

2. Recoil (radiation pressure),

3. transition saturation (stimulated emission),

and we suggest ways of mitigating them. Larmor precession is powerful enough to thwart optical pumping if the angle between the beam and the field lines is large (Drummond 2007), (Moussaoui 2009). The average 50-kHz redshift that one incurs per spontaneous emission due to atomic recoil can lead to spectral hole burning (depopulation of the respective atoms velocity group) in very bright single-frequency LGS (bandwidth $<10 \mathrm{MHz}$, Hillman (2008)), but it also offers the opportunity of "snowplowing" the sodium population towards higher velocities in the laser beam direction, requiring continuous blueshifting (chirping) of the laser as first suggested by Bradley (1992), at the expense of laser complexity. Ultimately, at high spectral irradiance, stimulated emission becomes relevant, limiting spontaneous emission. Photons from stimulated emission are emitted straight into space, hence becoming useless for LGS.

There is some ambiguity in the sodium LGS community about the term "saturation", for it is sometimes used to describe only transition saturation, and sometimes the depopulation of the $F=2$ upper ground state towards $F=1$, known as downpumping. Once an atom is in the $F=1$ state, it can only be excited if the $\mathrm{D}_{2} \mathrm{~b}$ line is pumped as well, which is known as repumping (either by allocating 10-20\% of the laser power to the $\mathrm{D}_{2} \mathrm{~b}$ line, or by widening a single laser line to $\geq 2 \mathrm{GHz}$ ). Downpumping in the absence of repumping becomes more severe with increasing laser irradiance and reduces the return flux long before the onset of stimulated emission, hence true transition saturation. Spinexchange collisions can bidirectionally exchange populations between the ground states and within them (transitions between the $(F=1, m= \pm 1)$ and $(F=2, m= \pm 2)$ ground substates are particularly strong). Repumping has already been experimentally demonstrated to be able to boost the LGS return flux by a factor of 1.6, (Telle 2008 finds a factor 2.4 through Bloch simulations), using two separate laser beams at different frequencies), 
and we show in this paper that more than a factor of 3 can be achieved.

To date, the most powerful sodium LGS system is installed at the Starfire Optical Range (SOR) at the Kirtland Air Force Base near Albuquerque, New Mexico, (Denman 2006a), fed by a single-frequency $(10-\mathrm{kHz}$ linewidth) $\mathrm{cw}$ laser that emits $50 \mathrm{~W}$ with circular or linear polarization. Due to its location, the median atmospheric seeing at SOR is significantly worse than at most sites of large astronomical telescopes, causing enlarged LGS spots in the mesosphere, and consequently limiting the irradiance. The upcoming generation of civilian 20-W-class LGS in astronomy, such as for the Adaptive Optics Facility of UT4 of the $\mathrm{VLT}^{2}$, is therefore expected to venture into unprecedented mesospheric irradiance regimes (much higher laser irradiances have of course been applied to gas cells, albeit at higher gas pressures). Next-generation lasers will emit circularly polarized $\mathrm{cw}$ radiation, use repumping, have a linewidth of a few $\mathrm{MHz}$, and are expected to return on the order of $10 \times 10^{6}$ photons $/ \mathrm{s} / \mathrm{m}^{2}$ on the ground. Quantifying and optimizing their return flux vs. the laser parameters is one of the principal purposes of this paper.

So far, we have only focused on photon return flux. What is really desired when designing AO systems, though, is a bright guide star, hence high luminosity concentrated in a small spot size. Compared to the uplink laser irradiance in the sodium layer, the above mentioned saturation effects spatially broaden the LGS return fluorescence distribution by emphasizing the low irradiance regions and dimming the peaks. We can show using physical-optics simulations, Holzlöhner (2008), that this effect increases the instantaneous spot sizes on a wavefront sensor by about $0.1^{\prime \prime}$, hence it is not negligible. At large angles between the laser beam and the geomagnetic field, downpumping can be mitigated by repumping.

In this work, we present a Bloch-equation method that models any alkali atom taking into account spontaneous and stimulated emission, Larmor precession due to the geomagnetic field, arbitrary elliptical light polarization, recoil, on the order of 100 coupled velocity groups with velocity-changing collisions and spin exchange, finite atomic dwell time in the beam (atom replacement), arbitrary laser bandwidth, and repumping. We neglect nonlinear Zeeman shifts and hyperfine coherences since we found them to have a small effect on the result. In contrast to the above cited Bloch simulation publications, we directly compute the steady state solution, which is more efficient than time domain solutions (a single run takes about $2 \mathrm{~s}$ on a modern PC). The program is written in Mathematica and based on the publicdomain Atomic Density Matrix package ${ }^{3}$.

In order to validate our results, we have also implemented a Monte Carlo rate-equation simulation called Exciter in Matlab, whose results we compare with the Bloch-equation method.

Section 2 describes the Bloch-equation method, Sect. 3 gives details about the simulation parameters, Sect. 4 presents the results, suggesting optimal cw sodium LGS formats, and we conclude in Sect. 5.

\footnotetext{
2 Adaptive Optics Facility (AOF) for the VLT, European Southern Observatory (ESO), http://www.eso.org/sci/facilities/ develop/ao/sys/.

3 Rochester, S. M., Atomic Density Matrix package for Mathematica, version 09.08.07 or later, available at http://budker.berkeley . edu/ADM/.
}

\section{Bloch equations}

In order to calculate the observed fluorescence from mesospheric sodium atoms, the evolution of the atoms is modeled using the optical Bloch equations for the atomic density matrix. The density matrix describes the statistical state of an ensemble of atoms in the state space of the $\mathrm{Na}_{2}$ transition. In order to account for atoms with different Doppler shifts, the density matrix is also considered to be a function of atomic velocity along the laser beam propagation direction. (An additional degree of freedom is included to account for laser line broadening as discussed below.) The calculation is semiclassical in the sense that while the atoms are treated quantum mechanically, the light fields are treated classically (the effect of spontaneous decay must be included phenomenologically since we do not apply field quantization). Because the density matrix describes all populations of, and coherences between, the 24 Zeeman sublevels making up the ground and excited states, the calculation describes, in principle, all saturation and mixing effects for essentially arbitrarily large optical and magnetic fields. (In practice, certain coherences in the system are negligible under our experimental conditions and can be neglected in order to increase the computational efficiency.)

In order to perform numerical calculations, the velocity dependence of the density matrix is discretized to describe an appropriate number $n_{\text {v.g. }}$ of velocity groups, each with a fixed longitudinal velocity. Because coherences between atoms with different velocities can be neglected, the complete density matrix $\rho$ can be thought of as a collection of $n_{\mathrm{v} . \mathrm{g} \text {. }}$ separate but coupled density matrices, each of dimension $24 \times 24$.

The evolution of the density matrix is given by a generalization of the Schrödinger equation:

$$
\frac{\mathrm{d}}{\mathrm{d} t} \rho=\frac{1}{i \hbar}[H, \rho]+\Lambda(\rho)+\beta,
$$

where $H=H_{0}+H_{\mathrm{E}}+H_{\mathrm{B}}$ is the total Hamiltonian, with $H_{0}$ the Hamiltonian for the unperturbed energy structure of the atom, $H_{\mathrm{E}}=-\boldsymbol{d} \cdot \boldsymbol{E}$ the Hamiltonian for the interaction of the electric dipole $\boldsymbol{d}$ of the atom with the electric field $\boldsymbol{E}$ of the light, $H_{\mathrm{B}}=-\mu \cdot \boldsymbol{B}$ the Hamiltonian for the interaction of the magnetic moment $\mu$ of the atom with the local magnetic field $\boldsymbol{B}$, $\hbar=h /(2 \pi)$ where $h=6.626 \times 10^{-34} \mathrm{Js}$ is Planck's constant, and the square brackets denote the commutator. The term $\Lambda$ in Eq. (1) represents phenomenological terms added to account for relaxation processes not described by the Hamiltonian (Budker 2002). In our case these relaxation processes include spontaneous decay (omitted from the Hamiltonian due to the semiclassical approximation), collisional spin relaxation ("S-damping") proportional to $S^{2} \rho-S \cdot(\rho S)$ (Happer 1987), and the exit of atoms from the light beam due to motion of the atoms and the beam. In addition, there are terms included in $\Lambda$ to describe changes in atomic velocity due to collisions and light-induced recoil, as well as an effective relaxation rate that describes dithering of the laser phase in order to simulate a finite bandwidth. These terms are described in more detail below. Each relaxation process described by $\Lambda$ includes a corresponding repopulation process, so that the trace over the density matrix for all velocity groups is conserved, corresponding to conservation of the total number of atoms. The repopulation process describing the entrance of atoms into the beam is independent of $\rho$ and so is written as a separate term $\beta$.

Velocity-changing collisions (vcc) are treated as hard collisions in which the velocity of the colliding atom is rethermalized in a Maxwellian distribution (no speed memory). The internal state of the atom is assumed to be unchanged. 
Light-induced recoil is described phenomenologically by causing a fraction $v_{\mathrm{r}} / \Delta v_{\mathrm{v} \text {.g. }}$ of the excited-state atoms in each velocity group to be transferred upon decay into the next higher velocity group. Here $v_{\mathrm{r}}$ is the recoil velocity and $\Delta v_{\mathrm{v} . \mathrm{g}}$. is the width of the particular velocity group. This model relies on the fact that $v_{\mathrm{r}}=2.9461 \mathrm{~cm} / \mathrm{s}$ (equivalent to a Doppler shift of $50.004 \mathrm{kHz}$ ) is much smaller than the typical value of $\Delta v_{\mathrm{v} . \mathrm{g}}$.

In order to simulate a finite bandwidth laser, a form of phase dithering is used (frequency or amplitude dithering can also be employed). To avoid resorting to a time-domain calculation, the dithering is implemented in the spatial domain: density matrices are written for two "regions" with light fields that are $\pi$ out of phase with each other, and relaxation terms are included that transfer the atoms between the regions (this doubles the size of the system of equations). The model is that of a laser beam with very fine "speckles" of different phases. The result is an effective laser spectrum of Lorentzian shape with a width proportional to the transfer rate between the regions. This method has been verified by comparison to a time-domain model (implemented for a nuclear-spinless system) in which the light frequency randomly changes with a Lorentzian distribution. Identical results from the two methods are obtained for the case in which the rate that the light frequency changes is faster than the natural decay rate.

Equation (1) supplies a linear system of differential equations for the density matrix elements, known as the optical Bloch equations. Thinking of $\rho$ as a column vector of $n_{v . g} \times 24^{2}$ density matrix elements, the Bloch equations can be written as $\dot{\rho}=A \rho+b$, where $A$ and $b$ are a matrix and vector, respectively, that are independent of $\rho$. The vector $b$ corresponds to $\beta$ and $A$ to the rest of the right-hand side of Eq. (1).

The laser light field has a frequency component tuned near the $\mathrm{D}_{2} F=2 \rightarrow F^{\prime}$ transition group $\left(\mathrm{D}_{2} \mathrm{a}\right)$, and may have an additional "repump" component tuned near the $F=1 \rightarrow F^{\prime}$ transition group $\left(\mathrm{D}_{2} \mathrm{~b}\right)$. Thus the matrix $A$ has components that oscillate at each of these frequencies. Under the rotating wave approximation (Corney 1977), the overall optical frequency is removed from $A$. However, the beat frequency between the two light-field components remains. This beat frequency can also be removed from the Bloch equations in our case: each frequency component interacts strongly with one transition group and very weakly with the other, so the weak coupling can be neglected for each transition. If, in addition, the small magnetic-field-induced mixing between the two hyperfine ground states is neglected, the beat frequency can be entirely removed from the evolution equations. This makes $A$ time-independent for $\mathrm{cw}$ light. To find the steady-state density matrix, we can set $\dot{\rho}=0$ and solve the linear system $A \rho=-b$. The vectors $\rho$ and $b$ have 322 elements per velocity group (576 if hyperfine states are not neglected), so that the sparse linear equation system has dimension 32 500-65000 in practice.

To solve the Bloch equations for a particular set of experimental parameters, we first choose an appropriate set of velocity groups. Since the signal is strongly peaked for atoms whose Doppler-shifted resonance frequency is near the light frequency, we can obtain more accurate results for a given number of velocity groups if narrower bins are used for resonant atoms, and wider for off-resonant. We have two methods for doing this.

The first method is to choose two fixed bin sizes, one narrow and one wide, and the number of narrow bins to cluster near each resonance. The wide bins are then used to take up the rest of the Doppler distribution. This method is useful when we don't know beforehand what the spectrum of the signal in velocity space is.

If we have an estimate of the spectrum (obtained using the first method), we can refine it using the second method, which takes advantage of this knowledge. We create a weighting function consisting of three terms: a constant term, which tends to make equal-sized bins, a term proportional to the spectrum, which makes more bins where the signal is large, and a term proportional to the magnitude of the second derivative of the signal, which makes more bins where the signal changes rapidly as a function of velocity. The bin sizes are then found by dividing the integral of the weighting function evenly into the chosen number of bins.

The linear system is solved using the implementation of the iterative BiCGSTAB method (stabilized biconjugate gradient, van der Vorst 1992) built-in to Mathematica. This is a Krylov subspace method in which an initial guess is improved by minimizing the residual over a subspace with dimension much smaller than that of the full system. The rate of convergence of the method is increased by pre-multiplication with a blockdiagonal preconditioner (approximate inverse of $A$ ), obtained by setting all terms that connect density matrix elements from different velocity groups to zero, and then inverting the block for each velocity group.

The fluorescent photon flux per solid angle emitted in a given direction can be found from the steady-state solution for $\rho$ as the expectation value of a fluorescence operator (Corney 1977).

\section{Simulation parameters}

\subsection{Determination of parameters}

Many physical constants and the atomic level diagram of $\mathrm{Na}$ have been summarized by Steck $^{4}$ and will not be repeated here. Table 1 lists further simulation parameters, and we walk through it to explain some quantities. We work in MKS units, except that we show magnetic field strength in Gauss $\left(1 \mathrm{G}=10^{-4} \mathrm{~T}\right)$ and atomic cross sections in squared centimeters.

The launched laser power $P$ equals the laser device output beam power, diminished by optical losses in the beam train and launch telescope (LT). The value of $I=46 \mathrm{~W} / \mathrm{m}^{2}$ is the " $50 \%$ power in the bucket" irradiance $I_{P / 2}$, as explained in the next subsection. We mostly deal with circularly polarized light (the ellipticity angle $\chi=\arctan (\varepsilon)= \pm \pi / 4$ denotes respectively LH/RH circular and $\chi=0$ linear polarization; $\pi / 2-2 \chi$ is the polar angle in the Poincaré sphere, and $\varepsilon$ is the ellipticity, hence the major-minor axes ratio of the polarization ellipse). The repumping fraction $q$ is the fraction of the total laser power allocated to the repump beam: the $\mathrm{D}_{2}$ a beam power is $(1-q) P$ and the power in the $\mathrm{D}_{2}$ b beam (tuned $\Delta f_{\mathrm{ab}}$ above the $\mathrm{D}_{2}$ a frequency) is $q P$.

The geomagnetic-field strength $B$ has a strong impact on the return flux. Its value varies considerably over the world and can be computed for different mesospheric altitudes using the International Geomagnetic Reference Field model ${ }^{5}$. Cerro Paranal in northern Chile, the location of the VLT and the reference site for this work $\left(24.6^{\circ} \mathrm{S}, 70.4^{\circ} \mathrm{W}\right)$, has $B \approx 0.23 \mathrm{G}$ at $92 \mathrm{~km}$ altitude, Mauna Kea (Hawaii) has $B \approx 0.35 \mathrm{G}$, and the Starfire Optical Range (Albuquerque, New Mexico) has $B \approx$ $0.48 \mathrm{G}$, about twice the field strength at Paranal.

The atmospheric transmission $T_{\mathrm{a}}$ at $589 \mathrm{~nm}$ was measured at Paranal to be 0.89 in photometric nights, (Patat 2004). We use a value that is $5 \%$ lower to account for higher aerosol levels. The

\footnotetext{
4 Steck, D. A., "Sodium D Line Data", version 2.1.1, 30 April 2009, http://steck.us/alkalidata/.

5 IGRF Release 2005 geomagnetic model online at NOAA website http://www .ngdc . noaa.gov/IAGA/vmod/.
} 
Table 1. Simulation parameters and their standard nominal values.

\begin{tabular}{|c|c|c|}
\hline Variable name & Symbol & Standard value \\
\hline \multicolumn{3}{|c|}{ Laser parameters } \\
\hline Launched laser power in air & $P$ & $20 \mathrm{~W}$ \\
\hline Mesospheric laser irradiance & $I$ & $46 \mathrm{~W} / \mathrm{m}^{2}$ \\
\hline Central $\mathrm{D}_{2}$ vacuum wavelength & $\lambda$ & $589.159 \mathrm{~nm}$ \\
\hline Polarization ellipticity angle & $\chi$ & $\pm \pi / 4$ (circular) \\
\hline Laser $F W H M$ linewidth & $\Delta f$ & 0 \\
\hline Repumping power fraction & $q$ & 0.12 \\
\hline Repumping frequency offset & $\Delta f_{\mathrm{ab}}$ & $1.7178 \mathrm{GHz}$ \\
\hline \multicolumn{3}{|c|}{ Atomic, atmospheric, and mesospheric parameters } \\
\hline Outer turbulence scale & $L_{0}$ & $25 \mathrm{~m}$ \\
\hline Geomagnetic field in mesosphere & $B$ & $0.228 \mathrm{G}$ \\
\hline One-way transmission at $\lambda$ at zenith & $T_{\mathrm{a}}$ & 0.84 \\
\hline Average mesospheric temperature & $T_{\mathrm{Na}}$ & $185 \mathrm{~K}$ \\
\hline Sodium centroid altitude (a.s.1.)* & $H_{\mathrm{Na}}$ & $92 \mathrm{~km}$ \\
\hline Sodium column density & $C_{\mathrm{Na}}$ & $4.0 \times 10^{13} \mathrm{~m}^{-2}$ \\
\hline Na beam dwell velocity & $v_{\gamma}$ & $38 \mathrm{~m} / \mathrm{s}$ \\
\hline Beam atom exchange rate & $\gamma_{\mathrm{ex}}$ & $1 /(6.0 \mathrm{~ms})$ \\
\hline $\mathrm{Na}-\mathrm{N}_{2}$ v.c.c. ${ }^{\dagger}$ cross section & $\sigma_{\mathrm{Na}-\mathrm{N}_{2}}$ & $0.71^{-14} \mathrm{~cm}^{2}$ \\
\hline $\mathrm{Na}-\mathrm{O}_{2}$ v.c.c. ${ }^{\dagger}$ cross section & $\sigma_{\mathrm{Na}-\mathrm{O}_{2}}$ & $0.70 \times 10^{-14} \mathrm{~cm}^{2}$ \\
\hline 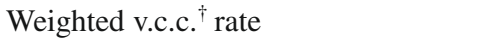 & $\gamma_{\mathrm{vcc}}$ & $1 /(35 \mu \mathrm{s})$ \\
\hline $\mathrm{Na}-\mathrm{O}_{2}$ spin exchange cross sect. at $T_{\mathrm{Na}}$ & $\sigma_{\mathrm{Na}-\mathrm{O}_{2}}^{\mathrm{S}}$ & $0.50 \times 10^{-14} \mathrm{~cm}^{2}$ \\
\hline Weighted spin-exchange rate at $T_{\mathrm{Na}}$ & $\gamma_{S}$ & $1 /(490 \mu \mathrm{s})$ \\
\hline \multicolumn{3}{|c|}{$\begin{array}{l}\text { Launch telescope }(\mathbf{L T}) \text { parameters } \\
\end{array}$} \\
\hline Zenith angle & $\zeta$ & $30^{\circ}$ \\
\hline LT altitude (a.s.1.)* & $H_{\text {tele }}$ & $2650 \mathrm{~m}$ \\
\hline LT aperture & $D$ & $40 \mathrm{~cm}$ \\
\hline $\mathrm{LT}$ beam radius $\left(1 / e^{2}\right)$ & $w$ & $0.36 D=14.4 \mathrm{~cm}$ \\
\hline Launched beam rms wavefront error & WFE & $100 \mathrm{~nm} \approx \lambda / 6$ \\
\hline Polar angle of $\boldsymbol{B}$ (laser $\| z$ ) & $\theta$ & $\pi / 2$ \\
\hline Azimuth of $\boldsymbol{B}$ (laser $\| z$ ) & $\phi$ & $\pi / 2$ \\
\hline
\end{tabular}

Notes. ${ }^{(*)}$ a.s.l. $=$ above sea level. ${ }^{(\dagger)}$ v.c.c. $=$ velocity-changing collision.

mesospheric temperature, as well as partial gas densities, can be derived using the MSISE-90 model $^{6}$.

Our values for the sodium layer centroid altitude $H_{\mathrm{Na}}$ and column abundance $C_{\mathrm{Na}}$ are obtained from studies taking place for over 30 years in São Paulo, (Simonich 1979; Moussaoui 2010), a site whose latitude differs only by one degree from the ESO Paranal Observatory. We believe that the sodium layer parameter statistics on the seasonal and daily variations are valid for Paranal.

Atomic collisions have a significant effect on the sodium states and hence on the LGS return flux. Since mesospheric sodium is rarefied (the total mass of global mesospheric sodium is about $600 \mathrm{~kg}$ ), $\mathrm{Na}-\mathrm{Na}$ collisions are negligible compared to $\mathrm{Na}-\mathrm{N}_{2}$ and $\mathrm{Na}-\mathrm{O}_{2}$ collisions. Most of these collisions are binary (collision of two molecules). One important effect of collisions is to change the velocity of the atoms, causing diffusion of optically pumped atoms in velocity space, sometimes called v-damping (Happer 1987). Since the masses of $\mathrm{N}_{2}$ and $\mathrm{O}_{2}$ molecules are comparable to that of $\mathrm{Na}$ atoms, one can assume that every collision completely randomizes their velocity. The collision rate of a gas of particle mass $M_{1}$ with another gas type of particle mass $M_{2}$ and number density $n_{2}$ is given by (Wright 2004)

$\gamma_{12}=n_{2} \sigma_{12} \sqrt{\frac{8 k_{\mathrm{B}} T}{\pi}\left(\frac{1}{M_{1}}+\frac{1}{M_{2}}\right)}$,

6 MSISE-90 atmospheric model online at NASA website http: //ccmc.gsfc.nasa.gov/modelweb/atmos/msise.html. where $k_{\mathrm{B}}=1.3807 \times 10^{-23} \mathrm{~J} / \mathrm{K}$ is Boltzmann's constant and $\sigma_{12}=\pi\left(r_{1}+r_{2}\right)^{2}$ is the collisional cross section with the effective particle radii $r_{1}, r_{2}$. Measuring these radii for velocitychanging collisions is difficult; here we just assume the Van-derWaals radii of $r_{\mathrm{Na}}=227 \mathrm{pm}, r_{\mathrm{N}_{2}}=250 \mathrm{pm}$, and $r_{\mathrm{O}_{2}}=245 \mathrm{pm}^{7}$, (Fishbane 2005). The effect of other gas species is negligible. With these numbers, we have, for example, $\sigma_{\mathrm{Na}-\mathrm{N}_{2}}=0.72 \times$ $10^{-14} \mathrm{~cm}^{2}$ and $\gamma_{\mathrm{Na}-\mathrm{N}_{2}}=3.98 \times 10^{-10} \mathrm{~cm}^{-3} \mathrm{~s}^{-1} \times n_{\mathrm{N}_{2}}=1 /(62.8 \mu \mathrm{s})$ at the sodium centroid $\left(n_{\mathrm{N}_{2}}=4.0 \times 10^{13} \mathrm{~cm}^{-3}\right)$.

The sodium layer has a median $F W H M$ thickness of $11.1 \mathrm{~km}$ and its median centroid lies at $H_{\mathrm{Na}}=92 \mathrm{~km}$ (Moussaoui 2010). The gas pressure decreases exponentially with altitude and the collision rate varies across the layer by about one order of magnitude, as shown by the MSISE-90 atmospheric model. We compute the mean collision rate of $\mathrm{Na}$ with $\mathrm{N}_{2}$ and $\mathrm{O}_{2}$, based on a table of $n_{\mathrm{N}_{2}}, n_{\mathrm{O}_{2}}$, and $T_{\mathrm{Na}}$ as functions of altitude, weighted by the assumed Gaussian sodium density distribution. The result, which we will use throughout this work, is $\gamma_{\mathrm{vcc}}=\gamma_{\mathrm{Na}-\mathrm{N}_{2}, \mathrm{O}_{2}}=$ $1 /(35 \mu \mathrm{s})$, which is three times higher than Milonni's (1999) assumption of $1 /(100 \mu \mathrm{s})$. Since the sodium abundance and layer thickness and altitude are highly variable and its profile often deviates significantly from Gaussian, one cannot expect a high accuracy in this parameter.

The other important relaxation mechanism beside $v$-damping is spin-exchange relaxation or S-damping (Happer 1987, Sect. 13), in particular for $\mathrm{Na}-\mathrm{O}_{2}$ collisions. Spin relaxation time constants have been measured between rubidium and metastable triplet helium (He*, Dmitriev 2008), as well as between rubidium and $\mathrm{H}_{2}, \mathrm{O}_{2}$, and $\mathrm{N}_{2}$, (Nagengast 1998) and sodium and various gases (Ramsey 1964; Kartoshkin 1998). A major difficulty with such measurements is that the overwhelming contribution to $\mathrm{S}$-damping of $\mathrm{Na}$ in the mesosphere is due to collisional spin exchange with $\mathrm{O}_{2}$, however, in gas cells $\mathrm{O}_{2}$ oxidizes $\mathrm{Na}$ quickly and hence this particular cross section is hard to determine experimentally. Theoretical calculations of the cross section involve Born-Oppenheimer molecular potential curves of doublet/quartet surfaces for $\mathrm{Na}-\mathrm{O}_{2}$, analogous to the singlet/triplet curves for $\mathrm{Na}-\mathrm{Na}$ and have not yet been carried out to our knowledge.

We estimate $\sigma_{\mathrm{Na}-\mathrm{O}_{2}}^{\mathrm{S}}=0.5 \times 10^{-14} \mathrm{~cm}^{2}$ at $185 \mathrm{~K}$, based on spin-exchange cross section measurements of $\mathrm{Na}-\mathrm{He}^{*}$ and $\mathrm{O}_{2}-$ He. However, only $1 / 2$ of this cross section is effective in our case (Dmitriev 2008, Eq. (3)), although the details of the cross-section calculation are under investigation at the moment. Setting $\sigma_{12}=\sigma_{\mathrm{Na}-\mathrm{O}_{2}}^{\mathrm{S}} / 2$ in Eq. (2), we obtain $\gamma_{S}=1 /(680 \mu \mathrm{s})$ at $92 \mathrm{~km}$ altitude, which is close to the value $\gamma_{S}=1 /(640 \mu \mathrm{s})$ that Milonni (1999) finds through fitting to experiment. Note, however, that Milonni's initial guess was $\sigma_{\mathrm{Na}-\mathrm{O}_{2}}^{\mathrm{S}}=1.0 \times 10^{-14} \mathrm{~cm}^{2}$, and he does not apply the scaling factor of $1 / 2$. Performing the same sodium density weighted averaging over altitude as above, we obtain $\gamma_{S}=1 /(490 \mu \mathrm{s})$, which will be used throughout this work. We will discuss the sensitivity of the Na return flux to variations in $\gamma_{S}$ and $\gamma_{\mathrm{vcc}}$ in the following section.

The rms lateral velocity $v_{\gamma}=d \gamma_{\mathrm{ex}}$ describes the sodium atom exchange into and out of the beam, where $d \approx 23 \mathrm{~cm}$ is the median FWHM mesospheric speckle diameter (see the following section), and $\gamma_{\mathrm{ex}}$ is the atom exchange rate. We assume that $v_{\gamma}$

\footnotetext{
7 Atomic beam scattering experiments yield significantly larger cross sections. We will provide details in a forthcoming publication. Online atomic radius list http://www.ccdc.cam.ac.uk/products/csd/ radii/.
} 
consists of four components that we sum in quadrature since in general they have uncorrelated directions:

(a) gas diffusion orthogonal to the beam;

(b) mesospheric wind orthogonal to the beam;

(c) beam wander caused by atmospheric turbulence; and

(d) LGS beam slewing due to star tracking.

Each of these contributions can be estimated:

(a) the diffusion coefficient of $\mathrm{Na}$ in air (mostly $\mathrm{N}_{2}$ ) can be calculated using the Chapman-Enskog formula (Cussler 1997), yielding $D_{\mathrm{Na}}=8.56 \times 10^{4} \mathrm{~cm}^{2} / \mathrm{s}$, close to Milonni's assumption of $D_{\mathrm{Na}}=1.0 \times 10^{5} \mathrm{~cm}^{2} / \mathrm{s}$. The effective lateral diffusion velocity across the laser speckle is hence $\sqrt{2 / 3} D / d=$ $30 \mathrm{~m} / \mathrm{s}$, where we apply the factor $\sqrt{2 / 3}$ since we consider only the diffusion orthogonal to the beam. Note that $D_{\mathrm{Na}}$ scales like $\sqrt{T_{\mathrm{Na}}} / n_{\mathrm{N}_{2}}$. At an altitude of about $104 \mathrm{~km}$ the mean free path of sodium exceeds $d$ and the atomic motion within the beam is no longer diffusive (the mean lateral ballistic velocity is then $370 \mathrm{~m} / \mathrm{s}$ );

(b) we use the horizontal wind model HWM07 which computes zonal and meridional winds at selected mesospheric altitudes and times (Drob 2008). We find a median horizontal wind speed magnitude at Paranal of $20 \mathrm{~m} / \mathrm{s}$. Nocturnal fluctuations by a factor of 2 are common;

(c) physical-optics simulations show an rms beam wander in the mesosphere of about $20 \mathrm{~cm}\left(\zeta=30^{\circ}\right)$, (Holzlöhner 2008), a value supported by analytical approximations (Andrews 2005, Ch. 12, Eq. (51)). The beam wanders on the time scale of $\tau_{\text {beam }}=d /$ (atmospheric wind speed) $\approx 23 \mathrm{~cm} / 10 \mathrm{~m} / \mathrm{s}=$ $23 \mathrm{~ms}$, leading to a beam wander velocity of $8.7 \mathrm{~m} / \mathrm{s}$;

(d) the tracking speed of the laser beam in the mesosphere at $\zeta=30^{\circ}$ is about $(2 \pi / 24 \mathrm{~h}) \times L=7.6 \mathrm{~m} / \mathrm{s}$, where $L=$ $X\left(H_{\mathrm{Na}}-H_{\text {tele }}\right) \approx 103 \mathrm{~km}$ is the line-of-sight distance to the guide star centroid with the geometrical length extension factor (airmass) $X=\sec (\zeta)$, equaling the secant of the zenith angle $\zeta$.

Summing these four velocities in quadrature yields $v_{\gamma}=38 \mathrm{~m} / \mathrm{s}$ and hence $\gamma_{\mathrm{ex}}=1 /(6.0 \mathrm{~ms})$.

\subsection{Mesospheric spot size}

Given a certain launched laser power, the spot size and shape determine the mesospheric irradiance and thus have a strong impact on the return flux, particularly in the presence of strong optical pumping and for small magnetic field polar angles $\theta$. The instantaneous mesospheric laser spot size has been simulated using physical optics (Holzlöhner 2008). Atmospheric turbulence produces a fast changing (timescale a few milliseconds) speckle pattern on the sky due to diffraction, where the speckles have a FWHM diameter of about

$d=\frac{L \lambda}{2 w}$,

and $w$ is the $1 / e^{2}$ irradiance radius of the laser beam at the projector. The number of speckles and their beam wander are governed by the seeing, and the pattern changes on the time scale of a few milliseconds. Note that the medium/long-term spot size as observed on the ground is significantly larger than $d$, and it does, in contrast to $d$, depend directly on the Fried length $r_{0}$ (Fried 1966), which is a size scale over which atmospheric phase variations remain below $2 \pi$. For a typical $r_{0}=5-25 \mathrm{~cm}$, assuming
Table 2. Mesospheric spot parameters for a $40 \mathrm{~cm}$ launch telescope under standard conditions $\left(P=20 \mathrm{~W}, \zeta=30^{\circ}\right)$

\begin{tabular}{lllll}
\hline \hline Seeing $\left({ }^{\prime \prime}\right)$ & WFE $(\mathrm{nm})$ & $I_{P / 2}\left(\mathrm{~W} / \mathrm{m}^{2}\right)$ & $F W H M_{\text {eff }}(\mathrm{cm})$ & $d(\mathrm{~cm})$ \\
\hline 0.6 & 70 & 79.1 & 30.0 & 22.5 \\
0.6 & 100 & 59.1 & 34.7 & 24.3 \\
1.0 & 70 & 43.5 & 40.4 & 23.8 \\
1.0 & 100 & 33.5 & 46.1 & 26.1 \\
\hline
\end{tabular}

an outer turbulence scale of $L_{0}=25 \mathrm{~m}$, there is a single strong central speckle and a couple of much weaker satellite speckles.

The LGS return flux depends in general nonlinearly on the mesospheric laser irradiance $I$, and the return light has to pass the turbulent atmosphere again on the downlink to reach the telescope; thus the irradiance distribution $I(x, y)$, as it would appear on a screen at the mesosphere, cannot be directly observed. To account for the effect of turbulence on the return light and characterize speckle pattern statistically, we collect statistics over many simulated realizations of $I(x, y)$ for different turbulence phase screens. We rewrite the usual optical power integral

$P\left(T_{\mathrm{a}}\right)^{X}=\int I \mathrm{~d} A=\int_{0}^{I_{\max }} I \frac{\mathrm{d} A}{\mathrm{~d} I} \mathrm{~d} I$

as an integral over irradiance up to the maximum value $I_{\max }$, where $A$ is area orthogonal to the beam and $\left(T_{\mathrm{a}}\right)^{X}$ is the atmospheric transmission along the line of sight (zenith angle $\zeta$ ). The quantity $\mathrm{d} A / \mathrm{d} I$ can be thought of as a histogram of mesospheric laser irradiance. We define $I_{P / 2}$ by

$\frac{P\left(T_{\mathrm{a}}\right)^{X}}{2}=\int_{0}^{I_{P / 2}} I \frac{\mathrm{d} A}{\mathrm{~d} I} \mathrm{~d} I=\int_{I_{P / 2}}^{I_{\max }} I \frac{\mathrm{d} A}{\mathrm{~d} I} \mathrm{~d} I$,

representing an irradiance of " $50 \%$ power in the bucket", which will be convenient to compute laser efficiencies later (note that usually $I_{\max } \gg 2 I_{P / 2}$ ). In order to model a realistic LGS beam scenario, we assume a $D=40 \mathrm{~cm} \mathrm{LT}$, a launched Gaussian beam of $1 / e^{2}$ (in irradiance) radius $w=0.72 D / 2=14.4 \mathrm{~cm}$, two different seeing values of $0.6^{\prime \prime}$ and $1.0^{\prime \prime}$ (site monitor seeing at $500 \mathrm{~nm}$ and zenith), and total wavefront errors (WFE) of the launched beam of $70 \mathrm{~nm} \mathrm{rms}(\approx \lambda / 8)$ and $100 \mathrm{~nm} \mathrm{rms}(\approx \lambda / 6)$. We simulate the actual mesospheric irradiance distribution and $I_{P / 2}$ using physical optics, and record the equivalent beam diameter $F W H M_{\text {eff }}$ that a single Gaussian spot of the same mesospheric power and same $I_{P / 2}$ would have, as well as the speckle size $d$, as in Holzlöhner (2008). Table 2 summarizes the results. The mean of $I_{P / 2}$ on lines 2 and 4 equals $46 \mathrm{~W} / \mathrm{m}^{2}$, which we will use as the reference irradiance in this work. The median values of $d$ in the last column are somewhat above the analytical prediction of Eq. (3) of $21.2 \mathrm{~cm}$, mostly due to the finite WFE.

\subsection{Flux quantities}

In the following, we define five flux quantities used in this article:

- our simulations compute the return flux per solid angle $\Psi$ as the number of photons per atom and per unit time spontaneously emitted in the direction of the launch telescope, as observed in the mesosphere, with units of photons/s/sr/atom; - in order to compare different laser formats at similar irradiances, one can divide $\Psi$ by the laser irradiance (in $\mathrm{W} / \mathrm{m}^{2}$ ) in the mesosphere to derive the specific atomic return flux $\psi=\Psi / I$ in units of photons $/ \mathrm{s} / \mathrm{sr} /$ atom $/\left(\mathrm{W} / \mathrm{m}^{2}\right)$, where area is measured in the mesosphere orthogonal to the beam. Most of our results are expressed in this quantity; 
- to compute the expected return flux on a receiver at a distance $L$ from the fluorescing atoms, we begin by integrating $\Psi$ over the receiver area $A$ orthogonal to the beam, subtending the solid angle $A / L^{2}$ to obtain the quantity $F_{\mathrm{m}}$;

- the photon flux on the detector $\Phi$ (unit photons $/ \mathrm{s} / \mathrm{m}^{2}$ ) at the sodium column abundance $C_{\mathrm{Na}}$ (unit atoms $/ \mathrm{m}^{2}$ ) equals

$\Phi=\frac{C_{\mathrm{Na}} X\left(T_{\mathrm{a}}\right)^{X} F_{\mathrm{m}}}{L^{2}}$.

The airmass $X$ appears in the numerator because the sodium column along the laser beam scales like the airmass (note that the distance $L$ grows by the same factor). We do not account for laser power depletion with increasing propagation distance in the sodium layer because only about $\sigma \times C_{\mathrm{Na}} \approx$ $10^{-15} \mathrm{~m}^{2} \times 4 \times 10^{13} \mathrm{~m}^{-2} \approx 4 \%$ of the laser photons interact with any sodium atoms under the standard conditions of Table 1, the other $96 \%$ travel unused into space;

- in order to compare LGS systems at similar powers but different laser formats, beam spot sizes in the mesosphere, atmospheric transmission, and zenith angle, we can divide $F_{\mathrm{m}}$ by the laser power in the mesosphere $P\left(T_{\mathrm{a}}\right)^{X}$ to arrive at the figure of merit quantity $s_{\mathrm{ce}}$ in units of photons $/ \mathrm{s} / \mathrm{W} /\left(\right.$ atoms $\left./ \mathrm{m}^{2}\right)$

$s_{\mathrm{ce}}=\frac{F_{\mathrm{m}}}{P\left(T_{\mathrm{a}}\right)^{X}}=\frac{\Phi L^{2}}{P\left(T_{\mathrm{a}}\right)^{2 X} C_{\mathrm{Na}} X}$,

where $\left(T_{\mathrm{a}}\right)^{X}$ appears quadratically in the denominator of the last term, accounting for both up- and downlink. The quantity $s_{\text {ce }}$ is hence the photon flux on the ground, corrected for its dependence on sodium centroid height and abundance, airmass, atmospheric transmission, and, to first order, launched laser power (since $s_{\text {ce }}$ depends strongly on $\theta$, we will only compute it for $\theta=\pi / 2$ in this work). We will compare LGS laser beam efficiencies based on $s_{\mathrm{ce}}$. Note that in our definition $s_{\mathrm{ce}}$ is not a slope efficiency (which would be proportional to $\partial F_{\mathrm{m}} / \partial P$ ); see also d'Orgeville (2000).

\section{Results}

We compute $\psi$ using the method and parameters described above. Using the standard conditions of Table 1, we obtain $\psi=258 \mathrm{ph} / \mathrm{s} / \mathrm{sr} /$ atom $/\left(\mathrm{W} / \mathrm{m}^{2}\right)$. In the following, we first provide some numerical validations including scans of $\psi(I)$, followed by optimization studies of $\psi$ when varying light polarization ellipticity angle $\chi$, repumping frequency offset $\Delta f_{\mathrm{ab}}$, repumping power fraction $q$, and laser linewidth $\Delta f$. Unless otherwise noted, the standard conditions of Table 1 apply.

\subsection{Some numerical validations}

Figure 1 shows a comparison between the analytical Na absorption cross sections given by Eqs. (10), (11) in (Milonni 1998) at $T=185 \mathrm{~K}$ (dashed red line, the sum of two Gaussians) and the simulated effective cross section

$\sigma=\frac{h v W}{I}$

where $v=c / \lambda$ is the center frequency of the $\mathrm{D}_{2}$ a line, with $c$ the vacuum speed of light, and $W$ is the actual rate of spontaneous emissions at irradiance $I$ (solid blue curve; the inverse of $W$ is known as the cycle time). The abscissa shows detuning of the (main) laser line from the $\mathrm{D}_{2}$ a line center. The agreement is excellent. We add the caveat that this agreement is necessary, but

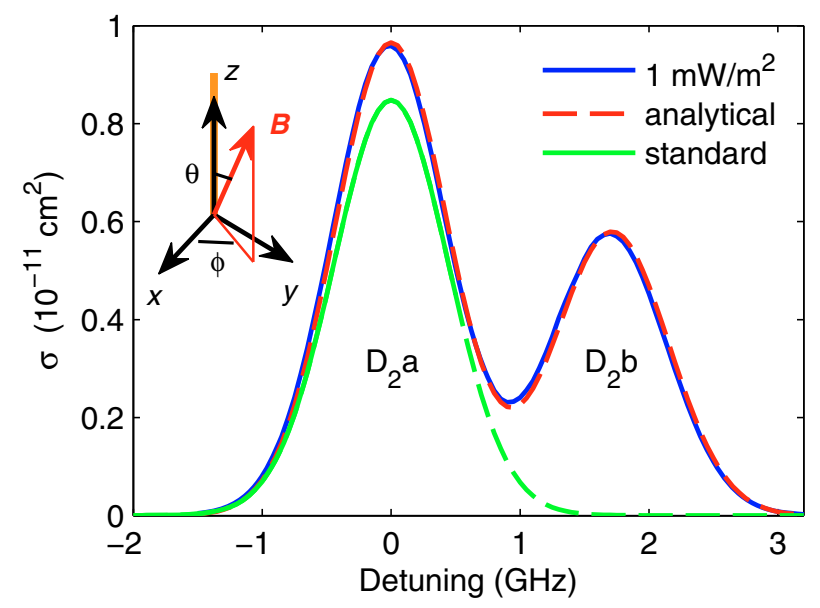

Fig. 1. Absorption cross section $\sigma$ vs. detuning from the $\mathrm{Na}_{2}$ a line center. Solid blue: simulation at $I=0.001 \mathrm{~W} / \mathrm{m}^{2}(q=0)$, dashed red: analytical cross section, green: simulation of effective cross section at standard conditions $\left(I=46 \mathrm{~W} / \mathrm{m}^{2}, q=0.12\right)$. Inset: reference coordinate system.

not sufficient to prove the validity of our algorithm since effects like Larmor precession, recoil, and stimulated emission have no effect at low irradiance as the $\mathrm{Na}$ atom is in thermal equilibrium.

The steady-state spontaneous emission rate $W$ cannot exceed $1 /(2 \tau)=1 /(32.5 \mathrm{~ns})$, and hence $\sigma$ tends to zero in the limit of infinite $I$. At $I=46 \mathrm{~W} / \mathrm{m}^{2}$ and $q=0.12$ (green curve), the reduction in $\sigma$ is still modest, however. We render the tail of the green curve towards the $\mathrm{D}_{2} \mathrm{~b}$ line center at $1.772 \mathrm{GHz}$ dashed, where our computational simplification of letting the " $\mathrm{D}_{2}$ a laser line" only excite the $\mathrm{D}_{2}$ a $\mathrm{Na}$ transitions, and analogously allowing the "repumping" line to only excite the $\mathrm{D}_{2} \mathrm{~b}$ transitions, whenever repumping is used $(q>0)$, breaks down. Once the detuning approaches the $\mathrm{D}_{2}$ b line center, this assumption obviously becomes invalid. By contrast, the blue curve was computed for $q=0$ and without using this simplification, and it is valid for any frequency offset.

The inset in Fig. 1 sketches the definition of the spherical angles $\theta$ and $\phi$ of the $\boldsymbol{B}$-vector in a coordinate system where the laser beam is projected along the $z$-axis. The major axis of the polarization ellipse for non-circular polarization is parallel to $x$.

Figure 2 shows the simulated atomic velocity distribution under standard conditions, except that Larmor precession and repumping are absent $\left(\theta=q=\gamma_{\mathrm{ex}}=0\right)$. The abscissa shows relative atomic velocity away from the receiver in frequency units (proportionality constant $1 / \lambda$ ). Recoil is usually associated with a frequency redshift, whereas we display the shift towards the blue. We choose this convention since a recoiled atom becomes resonant with a blueshifted laser line.

The blue curve represents the occupation histogram from the Monte Carlo rate-equation simulation Exciter after sampling $10^{6}$ different atomic velocities within the Doppler distribution ( $8.5 \times 10^{6}$ Monte Carlo steps simulating $26.6 \mathrm{~s}$ of physical time). The green curve is a Gaussian with the theoretical Doppler $F W H M$ width of $1.033 \mathrm{GHz}$ at $T_{\mathrm{Na}}=185 \mathrm{~K}$, representing the thermal equilibrium, and the magenta curve shows the occupation probability obtained from the Bloch-equation simulation. Finally, the cyan curve depicts for comparison the simulated return flux spectrum in the atomic frame, which in the present case of single-frequency excitation is close to the sodium natural line shape of a Lorentzian with a $F W H M$ of $1 /(2 \pi \tau)=9.8 \mathrm{MHz}$ (plotted at arbitrary vertical scale). 


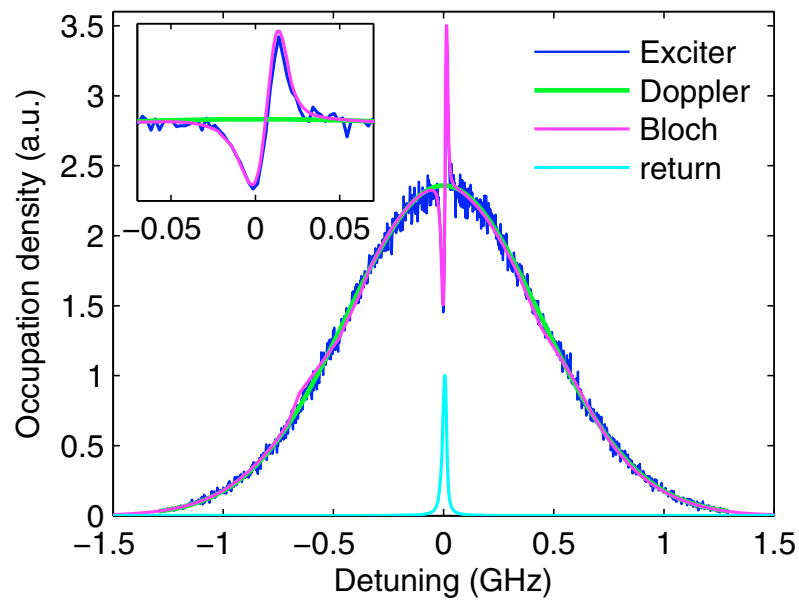

Fig. 2. Atomic velocity distribution under standard conditions (but $\theta=$ $q=0$ ), blue: Exciter, green: Doppler profile, magenta: Bloch equations, cyan: return light spectrum in the atomic frame. Inset: zoom on line center.

The effect of spectral hole burning is quite striking; in fact the occupation at the $\mathrm{D}_{2}$ a line center is depleted to $64 \%$ below the green Doppler curve. The atomic population, as seen from the telescope, is blue-shifted within about one velocity group. We observe that spectral hole burning due to recoil is in general exacerbated if repumping is applied, presumably due to the larger number of excitations per time. On the other hand, hole burning is mitigated when the laser bandwidth is extended at constant laser power because of the reduced spectral irradiance.

In Exciter, we increment the Doppler shift of the simulated atom by $50 \mathrm{kHz}$ each time a spontaneous emission occurs, which is correct on average. The agreement between Exciter and the Bloch equations is excellent, giving us confidence that the Gaussian velocity distribution and recoil are properly modeled.

At $I=46 \mathrm{~W} / \mathrm{m}^{2}$, Exciter simulates a spontaneous emission every $9.5 \mu$ s on average. Of all emissions, $10.2 \%$ are stimulated and $89.8 \%$ are spontaneous, and $70.6 \%$ of all atomic transitions occur along $(F=2, m=2) \leftrightarrow\left(F^{\prime}=3, m^{\prime}=3\right)$, indicating efficient optical pumping. We conclude that besides suffering from recoil, we are also starting to saturate this transition. At $I=100 \mathrm{~W} / \mathrm{m}^{2}, 17.9 \%$, and at $I=1000 \mathrm{~W} / \mathrm{m}^{2}, 55.2 \%$ of all emissions are stimulated, respectively. On average, one spontaneous emission occurs every $5.1 \mu$ s and $1.1 \mu$ s for these respective irradiances.

Figure 3 shows three semilogarithmic plots of $\psi(I)$ for the standard conditions of Table 1 unless noted otherwise, but neglecting exchange with thermal $\mathrm{Na}$ atoms outside the beam $\left(\gamma_{\mathrm{ex}}=0\right.$, which is not implemented in Exciter, but causes only a small difference). We hence simulate a single-frequency laser $(\Delta f \rightarrow 0)$ tuned to the peak of the $\mathrm{D}_{2}$ a line. Solid curves show $\psi$ obtained from the Bloch method, and the symbols indicate the results of Exciter. The green and red curves and symbols represent the case of laser beam parallel to the geomagnetic field $(\theta=0)$ with and without repumping, hence in the absence of Larmor precession, respectively. The blue and magenta curves and symbols are the same as green and red, respectively, but with the laser orthogonal to the field $(\theta=\pi / 2)$. Figure 3 a shows the case of circular, and Fig. $3 \mathrm{~b}$ that of linear polarization, the blue and magenta curves for $\theta=\phi=\pi / 2$ (note the difference in vertical scale).

We can make a number of interesting observations. First, the impact of the magnetic field is profound and reduces the return
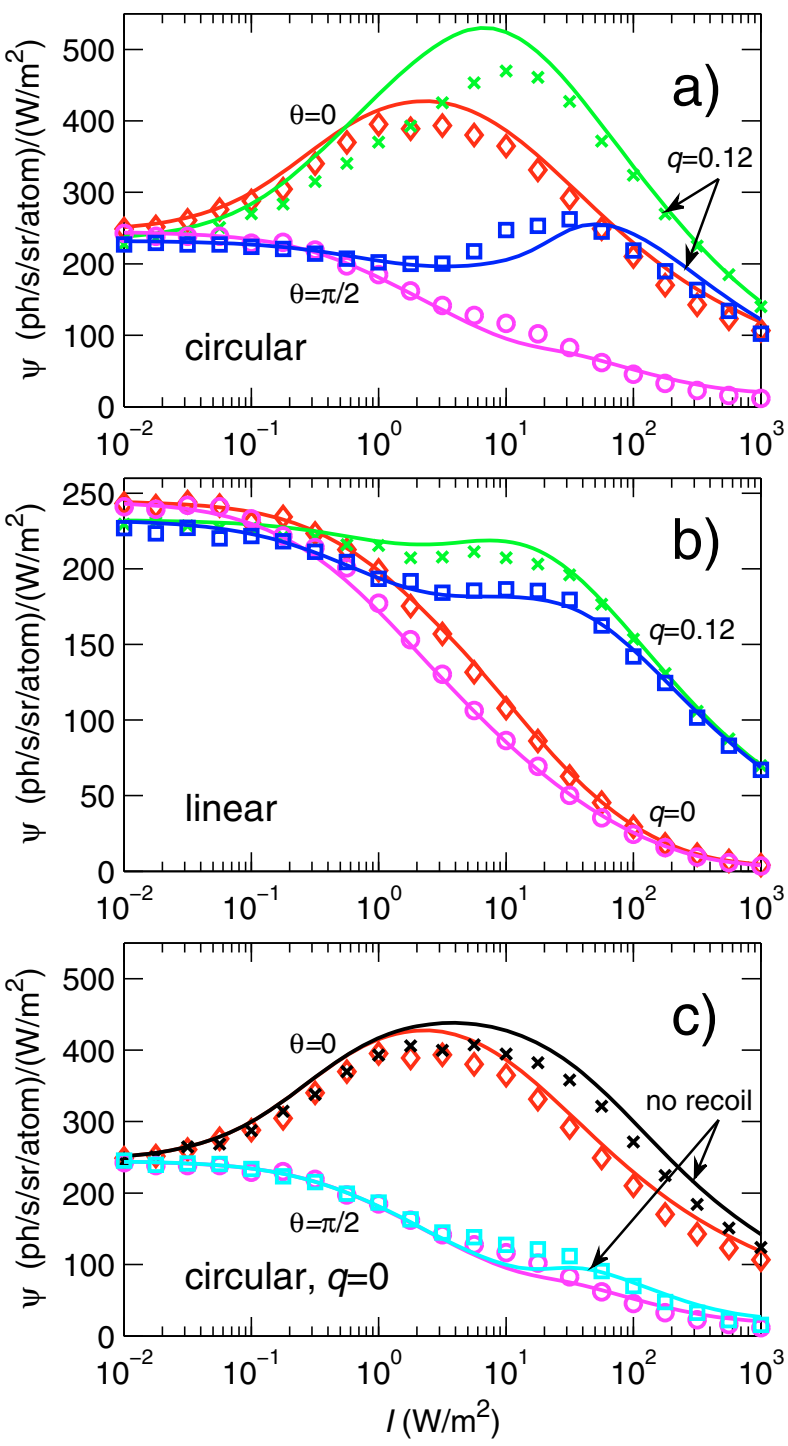

Fig. 3. Specific return flux $\psi(I)$. Lines: Bloch equations, symbols: Monte Carlo rate-equation simulation Exciter. a) Green, crosses: $\theta=$ $0, q=0.12$; red, diamonds: $\theta=0, q=0$; blue, squares: $\theta=\pi / 2, q=$ 0.12 (standard conditions); magenta, circles: $\theta=\pi / 2, q=0$ (all with circular polarization and $\gamma_{\mathrm{ex}}=0$ ). b) Same as a), but for linear polarization. c) Red and magenta lines, diamonds and circles: as in a); black, crosses and cyan, squares: same, respectively, without recoil.

flux strongly, at some irradiances by several times. At very low irradiance $\left(I=10^{-2} \mathrm{~W} / \mathrm{m}^{2}\right)$, the atom is in thermal equilibrium and all magnetic sublevels are nearly equally populated. Optical pumping sets in with increasing $I$ if using circular polarization, but Larmor precession is powerful enough to completely suppress it at $\theta=\pi / 2$, as evident from the monotonically falling magenta curve. Conversely, in the absence of Larmor precession $\psi$ strongly peaks near $I=2 \mathrm{~W} / \mathrm{m}^{2}$. The Larmor precession period equals

$\tau_{\mathrm{L}}=\frac{h}{\mu_{\mathrm{B}}\left|g_{F}\right| B}$

where $\mu_{\mathrm{B}}$ is the Bohr magneton and $g_{F}$ is the Landé factor that depends on $F$ ( $g_{F}=\mp 1 / 2$ for the $F=1,2$ sodium ground states, respectively). On Paranal, $\tau_{\mathrm{L}} \approx 6.3 \mu \mathrm{s}(159 \mathrm{kHz})$ and at SOR we find $\tau_{\mathrm{L}} \approx 3.0 \mu \mathrm{s}(333 \mathrm{kHz}, B=0.48 \mathrm{G})$. 
The green and blue curves portray the "healing" effect of repumping ( $q=0.12$, i.e., $12 \%$ of the laser power shifted to $\mathrm{D}_{2} \mathrm{~b}$ ). In the absence of Larmor precession, the peak is more pronounced and shifted to higher I (green). The highest impact occurs when the influence of the magnetic field is highest $(\theta=\pi / 2)$, where $\psi$ remains shallow until $80 \mathrm{~W} / \mathrm{m}^{2}$ and then decays gently. This finding refutes the often heard notion in the LGS community that "one should always stay away from the circular polarization saturation irradiation $\left(I_{\text {sat,circular }}=62.6 \mathrm{~W} / \mathrm{m}^{2}\right.$ to avoid saturation".

At very low $I$, repumping is ineffective and even slightly decreases the return flux due to the smaller cross section of the $\mathrm{D}_{2} \mathrm{~b}$ transition. At high $I$, repumping can readily compensate the degrading effect of Larmor precession; in its absence, $\psi$ decays to zero. For linear polarization, this decay even occurs for $\theta=0$ (red curve) due to depopulation of the upper ground state. Conversely, circular polarization "rescues" many atoms into the $(S, F=2, m= \pm 2) \leftrightarrow\left(P, F^{\prime}=3, m^{\prime}= \pm 3\right)$ transition, in which they are safe from downpumping.

Note that besides increasing the return flux by several times, a flatter function $\psi(I)$ also leads to smaller observed spot sizes since spatial power-broadening is reduced, a very welcome bonus.

The agreement between the Bloch calculation and Exciter is very good in almost all cases, given the completely different nature of the two methods, with the Bloch code usually yielding the higher values of $\psi$. Exciter finds the magnetic resonance bump at smaller $I$ due to difficulties with the proper simulation of two concurrent effects on similar, but not equal, time scales in our Monte Carlo rate-equation. Also the agreement between the green curve and the green crosses is somewhat poor around $I=0.2-20 \mathrm{~W} / \mathrm{m}^{2}$, presumably due to the simplified way in which Exciter models S-damping and/or the absence of coherences.

Figure $3 \mathrm{c}$ shows the impact of recoil. The red and magenta curves and symbols are as in subplot a), while black and cyan crosses and squares denote the same, respectively, but neglecting recoil. The magnetic resonance is better visible in the cyan than in the magenta curve. Recoil leads to a significant reduction in $\psi$ above $2 \mathrm{~W} / \mathrm{m}^{2}$.

Figure $4 \mathrm{a}$ shows $\psi$ as a function of the Larmor frequency $1 / \tau_{\mathrm{L}}$ (blue curve) at $I=1 \mathrm{~W} / \mathrm{m}^{2}$. The red dashed curve, overlapping the solid blue curve, is a fit function composed of the sum of two Lorentzians of different widths, centered at the origin, plus a constant term. The (half-)width of the narrower Lorentzian $\left(517 \mathrm{~Hz}=0.25 \gamma_{S}\right)$ is determined by the S-damping resonance, and that of the broader Lorentzian $\left(17.9 \mathrm{kHz}=0.63 \gamma_{\mathrm{vcc}}\right)$ by the velocity-changing collision rate, and the widths change proportionally when varying $\gamma_{S}$ or $\gamma_{\mathrm{vcc}}$. However, both resonances are somewhat power-broadened. The geomagnetic field is strong enough at $I=1 \mathrm{~W} / \mathrm{m}^{2}$ to place us on the lowest terrace of the blue curve, as indicated by the vertical lines. The blue diamonds show the result of Exciter for comparison, also exhibiting the terraces.

Figure $4 \mathrm{~b}$ shows the corresponding relative populations of the five $S, F=2$ upper ground state sublevels $(m=-2 \ldots 2)$, where the solid lines indicate Bloch equations and dotted lines Exciter. For $1 / \tau_{\mathrm{L}}>300 \mathrm{~Hz}$, the sublevel populations collapse to the same value due to Larmor-induced sublevel mixing. Conversely, for $1 / \tau_{\mathrm{L}}<300 \mathrm{~Hz}$, the populations diverge and the laser pumps the $m=2$ sublevel (blue) most strongly. When increasing the irradiance to $I=46 \mathrm{~W} / \mathrm{m}^{2}$ (not shown in the plot), the curves in b) would look similar, but the divergence point shifts upward to $\approx 1 \mathrm{kHz}$. Furthermore, when plotting the

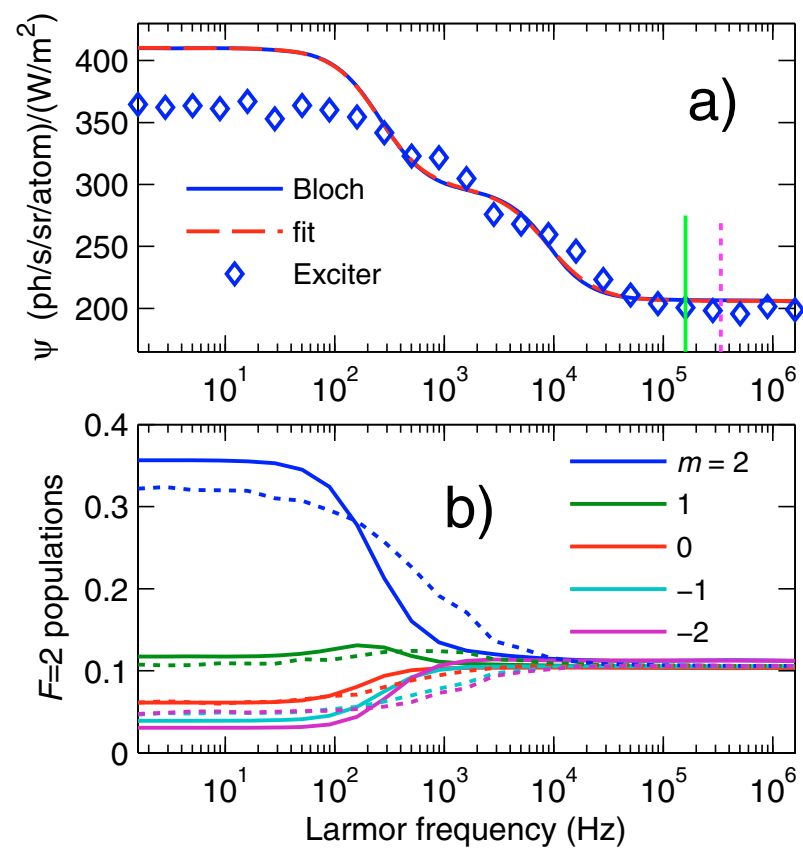

Fig. 4. Magnetic field impact. a) Blue curve: $\psi$ as a function of the Larmor frequency $1 / \tau_{\mathrm{L}}$ at $I=1 \mathrm{~W} / \mathrm{m}^{2}$. Red dashed curve (almost obscuring the blue curve): fit (sum of two Lorentzians). Blue diamonds: Exciter. Solid green (dashed magenta) lines: $1 / \tau_{\mathrm{L}}$ at Paranal (SOR). b) $F=2$ fractional sublevel populations, solid (dashed) curves: Bloch (Exciter) simulation.

Table 3. Sensitivity of $\psi$ to a $1 \%$ perturbation in some simulation parameters under standard conditions.

\begin{tabular}{|c|c|c|}
\hline Name of perturbed variable & Symbol & $\psi$ change $(\%)$ \\
\hline Mesospheric laser irradiance & $I$ & 0.025 \\
\hline Geomagnetic field in mesosphere & $B$ & -0.33 \\
\hline Average mesospheric temperature & $T_{\mathrm{Na}}$ & -0.48 \\
\hline Beam atom exchange rate & $\gamma_{\mathrm{ex}}$ & 0.0025 \\
\hline Weighted v.c.c. ${ }^{*}$ rate & $\gamma_{\mathrm{vcc}}$ & 0.15 \\
\hline Weighted spin-exchange rate at $T_{\mathrm{Na}}$ & $\gamma_{S}$ & 0.013 \\
\hline Recoil frequency $(50 \mathrm{kHz})$ & & -0.24 \\
\hline
\end{tabular}

Notes. ${ }^{(*)}$ v.c.c. $=$ velocity-changing collision.

sublevel populations at $I=46 \mathrm{~W} / \mathrm{m}^{2}$ as a function of $\theta$, one observes that the magnetic field at Paranal is just strong enough to collapse the populations for $\theta \rightarrow \pi / 2$. In other words, by scanning $\theta$ across the sky with a narrow-band 20-W-class laser, we can observe sodium excitation all the way from full optical pumping to no pumping at all, leading to a severe return flux penalty, as shown in the following figures.

Table 3 lists the sensitivity of $\psi$ w.r.t. a $1 \%$ perturbation in some simulation parameter $p$, more precisely $\psi(1.01 p) / \psi(p)-1$, where all other parameters are those of Table 1 . A value of $b \%$ in the last table column thus indicates that $\psi(p) \propto p^{b}$ in some range around the chosen value of $p$. We have selected only those parameters that we will not study in greater detail in the following subsections (except $I$ ). In addition, we have excluded those parameters around which $\psi$ is stationary $\left(\psi^{\prime}(p)=0\right)$, and also those parameters whose influence on the observed return flux is obviously linear, such as $C_{\mathrm{Na}}$.

From Table 3 , we notice that with the parameters of Table 1 the sensitivity to changes in $I$ is small. A comparison with Fig. 3b (dashed blue curve) shows that $\psi(I)$ is very shallow near $I=46 \mathrm{~W} / \mathrm{m}^{2}$ (the slope is actually weakly positive due to the 
magnetic resonance bump), meaning that $\Psi(I)=\psi I \propto I$, i.e. the absolute photon return grows linearly with the irradiance. The dependence of $\psi$ on $B$, however, is strong: Switching from $B=0.23$ (Paranal) to $B=0.48$ (SOR, factor 2.11) decreases $\psi$ by a factor of 0.76 ! The influence of temperature is significant as well since the width of the velocity distribution scales like $T_{\mathrm{Na}}^{1 / 2}$, and conversely its normalization (the number of $\mathrm{Na}$ atoms per velocity group) scales like $T_{\mathrm{Na}}^{-1 / 2}$. However, increasing $T_{\mathrm{Na}}$ from $185 \mathrm{~K}$ to $200 \mathrm{~K}$, which may be a typical seasonal variation, reduces $\psi$ by only $3.7 \%$, and hence common temperature variations do not directly influence $\psi$ much (we note that temperature influences the sodium abundance). Both $\gamma_{\mathrm{ex}}$ and $\gamma_{S}$ have a small influence on $\psi$, mainly due to the presence of repumping, as Milonni (1999) has also noted (for $q=0$, the sensitivities are $0.049 \%$ and $0.56 \%$, respectively).

We can draw two lessons here: There is little point in spending much effort trying to improve the spin-exchange cross section $\sigma_{\mathrm{Na}-\mathrm{O}_{2}}^{\mathrm{S}}$ estimate, and, secondly, repumping makes $\psi$ more robust towards some parameter variations, leading to higher stability in numerical simulations and possibly also in experiment. The variation of $\psi$ with $\gamma_{\mathrm{vcc}}$ is much larger than with $\gamma_{S}$, and at first sight surprisingly, the derivative is positive. We explain the positivity by the fact that collisions mitigate spectral hole burning due to recoil (neglecting recoil, the derivative is $-0.089 \%$ ). Finally, we have constants and $\lambda$ (more precisely $h /\left(m_{\mathrm{Na}} \lambda^{2}\right)=$ $50.0 \mathrm{kHz}$, with the atomic mass of $\mathrm{Na} m_{\mathrm{Na}}=3.819 \times 10^{-26} \mathrm{~kg}$ ), in order to demonstrate the importance of proper recoil modeling at $I>1 \mathrm{~W} / \mathrm{m}^{2}$.

In the following subsection, we will show that the laser parameters of Table 1 are close to optimal, given the other conditions of the table. We focus on the case $\theta=\pi / 2$ where the laser beam is directed orthogonal to the geomagnetic field (the worst case, for which LGS lasers must be designed to achieve a given return flux requirement).

\subsection{Optimization of $\psi$}

\subsubsection{Polarization ellipticity angle}

Figure 5 plots $\psi$ as a function of the laser polarization ellipticity angle $\chi$. To our knowledge, this is the first publication to discuss $\psi(\chi)$ for LGS. The axis limits \pm 1 of the quantity $4 \chi / \pi$ denote circular polarization (the poles of the Poincaré sphere; in our convention, $4 \chi / \pi=+1$ pumps towards increasing $m$ ), and $\chi=0$ indicates linear polarization. The curves pertain to different combinations of $\theta$ and $\phi$, the polar angle and azimuth of the $\boldsymbol{B}$ vector in a system where the laser points along $z$ (note that for $\theta=0$, the azimuth $\phi$ becomes meaningless at any light polarization. Conversely, when using any laser that is not purely circular polarized, $\phi$ does influence the return flux, which is often forgotten. When using purely linear polarized light, $\theta=\pi / 2, \phi=0$ is equivalent to setting $B=0$ within our convention, while $\phi=\pi / 2$ induces the strongest effect from Larmor precession.)

The cosine-like shape of $\psi(\chi)$ presents an initially gradual decrease of the return flux from $4 \chi / p i= \pm 1$, meaning that high polarization purity is not required when pumping the sodium with circularly polarized light. If one is willing to accept a decrease in $\psi$ of $5 \%$ along the solid red curve, it is enough to maintain $4|\chi| / \pi \geq 0.8$, i.e., down to an ellipticity angle of $|\chi|=36^{\circ}$. The polarization extinction ratio (PER) in $\mathrm{dB}$ is given by PER $=-20 \log _{10} \tan |\chi|$ and assumes the values of 0 and $\infty$ for circular and linear polarization, respectively. The condition

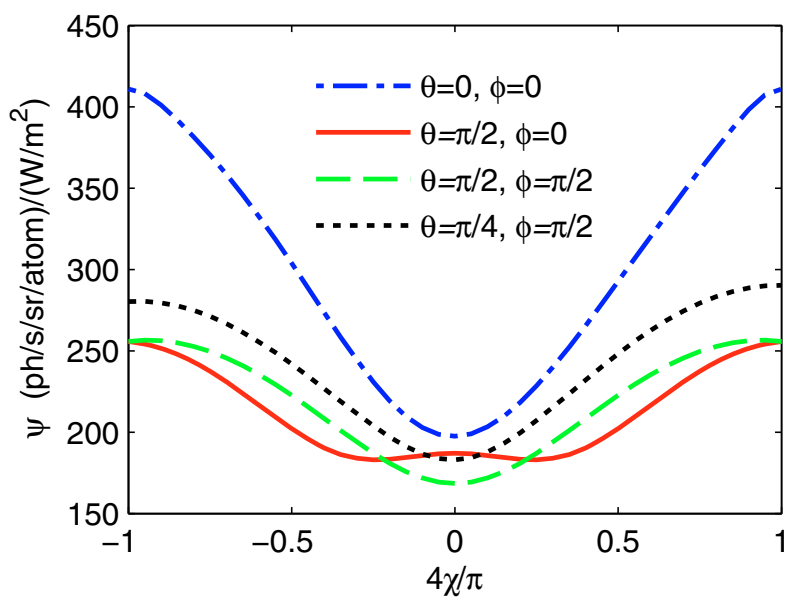

Fig. 5. $\psi$ as a function of laser polarization ellipticity angle $\chi$, where $4 \chi / \pi= \pm 1(0)$ marks circular (linear) states. The curves indicate different combinations of the magnetic field polar angle $\theta$ and azimuth angle $\phi$. Note that $\psi(\chi)$ is highest for circular polarization in all cases.

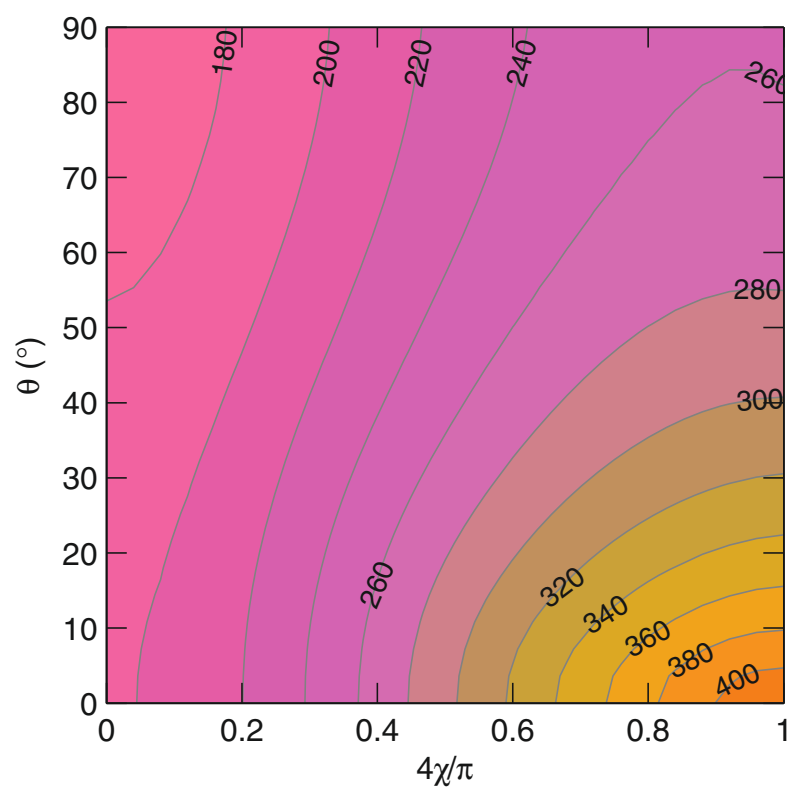

Fig. 6. Contour plot of $\psi(\chi, \theta)$ in $\mathrm{ph} / \mathrm{s} / \mathrm{sr} /\left(\mathrm{W} / \mathrm{m}^{2}\right)$ for magnetic azimuth $\phi=\pi / 2$ at $I=46 \mathrm{~W} / \mathrm{m}^{2}$.

of $4|\chi| / \pi \geq 0.8$ implies PER $\leq 6.4 \mathrm{~dB}$. This insensitivity is good news for the design of launch telescope optics.

Figure 6 shows a contour plot of $\psi(\chi, \theta)$ in $\mathrm{ph} / \mathrm{s} / \mathrm{sr} /\left(\mathrm{W} / \mathrm{m}^{2}\right)$ for $\phi=\pi / 2$ (worst case) under standard conditions. Current LGS systems, even SOR's FASOR laser, operate at lower " $P / 2$ " irradiances than $I_{P / 2}=46 \mathrm{~W} / \mathrm{m}^{2}$ and/or at linear polarization, for which $\psi(\theta)$ varies slowly. At higher $I$ and circular polarization, $\psi(\theta)$ is a steeper function (along the right vertical plot edge). For $\theta>45^{\circ}$, also $\psi(\chi)$ varies slowly near $4|\chi| / \pi=1$, indicating again that the circular polarization purity does not have to be very high.

\subsubsection{Repumping frequency offset}

Figure 7 shows $\psi$ as a function of $\Delta f_{\mathrm{ab}}$, the repumping frequency offset between $\mathrm{D}_{2} \mathrm{a}$ and $\mathrm{D}_{2} \mathrm{~b}$, relative to $1.7178 \mathrm{GHz}$, for two single-frequency laser lines and $q=0.12$ at $I=1 \mathrm{~W} / \mathrm{m}^{2}$ (dashed green), $I=10 \mathrm{~W} / \mathrm{m}^{2}$ (dash-dotted blue), and $I=46 \mathrm{~W} / \mathrm{m}^{2}$ (solid 
R. Holzlöhner et al.: Optimization of cw sodium laser guide star efficiency

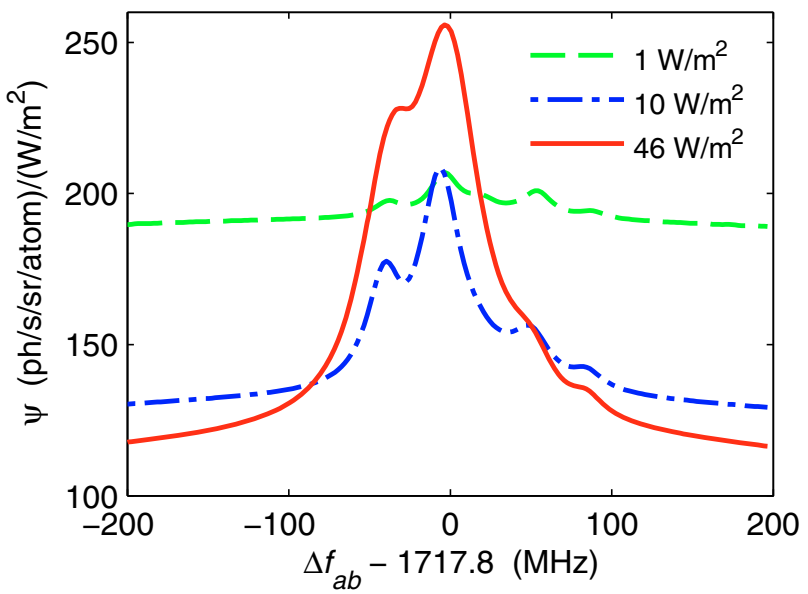

Fig. 7. $\psi$ as a function of repumping laser line frequency offset $\Delta f_{\mathrm{ab}}$ at $I=1 \mathrm{~W} / \mathrm{m}^{2}$ (dashed green), $I=10 \mathrm{~W} / \mathrm{m}^{2}$ (dash-dotted blue), and $I=46 \mathrm{~W} / \mathrm{m}^{2}$ (solid red).

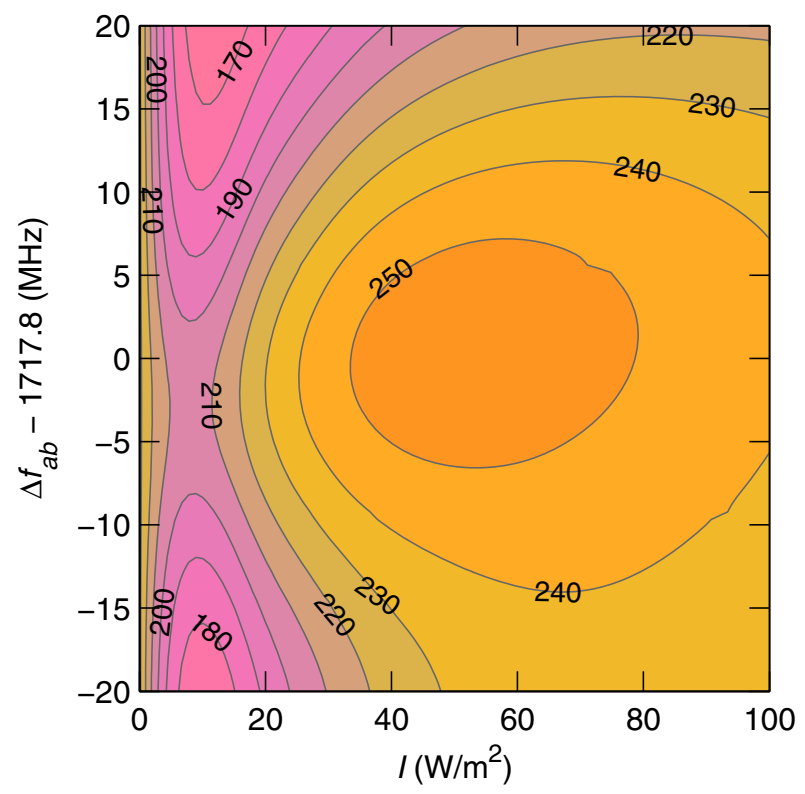

Fig. 8. Contour plot of $\psi\left(I, \Delta f_{\mathrm{ab}}\right)$ in $\mathrm{ph} / \mathrm{s} / \mathrm{sr} /\left(\mathrm{W} / \mathrm{m}^{2}\right)$.

red). The repumping from $(S, F=1) \rightarrow(S, F=2)$ requires excitation to the states $\left(P, F^{\prime}=1\right)$ or $\left(P, F^{\prime}=2\right)$ since no transitions $(S, F=1) \rightarrow\left(P, F^{\prime}=3\right)$ are allowed.

Figure 8 is a contour plot of $\psi\left(I, \Delta f_{\mathrm{ab}}\right)$, zooming into the region of $\Delta f_{\mathrm{ab}}=1717.8 \pm 20 \mathrm{MHz}$. In order to harness the full improvement of repumping, one needs to adjust $\Delta f_{\mathrm{ab}}$ with a precision of a few $\mathrm{MHz}$, particularly when at intermediate irradiance $\left(I \approx 10 \mathrm{~W} / \mathrm{m}^{2}\right)$. At larger irradiance, power-broadening washes out the peak (a laser beam has irradiances $0 \leq I \leq I_{\max }$ in the mesosphere, located along a horizontal line in the diagram). The tolerance in $\Delta f_{\mathrm{ab}}$ is so narrow because repumping is most efficient if a given $\mathrm{Na}$ atom that has been downpumped to the $F=1$ lower ground state can be reexcited before its velocity changes due to a collision, for which the frequency offset of the repumping laser line should lie between the $(S, F=$ $1) \rightarrow\left(P, F^{\prime}=1,2\right)$ transition frequencies minus the $(S, F=$ $2) \rightarrow\left(P, F^{\prime}=3\right)$ transition frequency that the main laser line is tuned to, i.e., close to $1717.8 \mathrm{MHz}$.

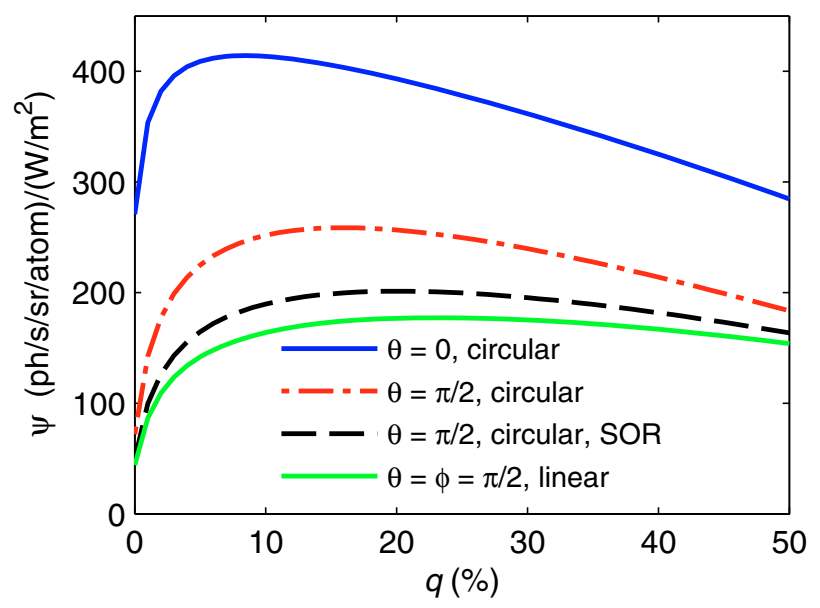

Fig. 9. $\psi$ as a function of repumping power fraction $q$ for different field angles $\theta$. Dash-dotted red curve: standard conditions, solid blue: $\theta=0$ (circular polarization, no Larmor precession), dashed black: standard conditions, but $B=0.48 \mathrm{G}$ (SOR), solid green: linear polarization $(\theta=$ $\phi=\pi / 2)$.

\subsubsection{Repumping power fraction}

Figure 9 portrays the calculated $\psi(q)$ for different magnetic field polar angles $\theta$ and field strengths $B$. The dash-dotted red curve is for standard conditions of Table 1, the solid blue curve for $\theta=0$ (i.e., circular polarization and no Larmor precession), the dashed black curve is for standard conditions, but with the magnetic field at SOR, and the solid green curve is for linear polarization (worst case $\theta=\phi=\pi / 2$ ).

All curves peak around $q=8-25 \%$, and the maximum occurs at larger $q$ the stronger the magnetic field effect is. Note that $\psi(q)$ peaks at $q>0$ even in the absence of Larmor precession (blue curve) since there is always a chance that atoms decay to the lower ground state. Moreover, $\psi(q)$ is very steep at $q=0$, rewarding even the weakest repumping, which is the reason why broadline lasers $(\approx 2 \mathrm{GHz})$ often do reasonably well, despite their poor spectral overlap with $\sigma(v)$ (see Fig. 1). Our simulations, however, indicate that a narrow-band laser with $12 \%$ repumping beats the return flux of a single-line $2 \mathrm{GHz}$-linewidth laser by a factor of 3.7 under standard conditions (see next subsection).

For circular polarization we find $\psi(q=0.12) / \psi(q=0) \approx 3.5$ at $I=46 \mathrm{~W} / \mathrm{m}^{2}$, underlining again that repumping is extremely beneficial, in particular in future LGS systems involving high irradiance.

Figure 10 shows six semilogarithmic contour plots of $\psi(I, q)$ (note the different color scales of the plots). The blue crosses indicate the point $\left(I=46 \mathrm{~W} / \mathrm{m}^{2}, q=0.12\right)$. Plot a) exhibits $\psi$ for linear polarization at Paranal for $\theta=\phi=\pi / 2$, which only varies weakly with $B$. At the blue cross, $\psi=169 \mathrm{ph} / \mathrm{s} / \mathrm{sr} /\left(\mathrm{W} / \mathrm{m}^{2}\right)$ (for $q=0$, it is only $44 \mathrm{ph} / \mathrm{s} / \mathrm{sr} /\left(\mathrm{W} / \mathrm{m}^{2}\right)$ !). Plot $\mathrm{b}$ ) shows the same for circular polarization $\left(\psi=258 \mathrm{ph} / \mathrm{s} / \mathrm{sr} /\left(\mathrm{W} / \mathrm{m}^{2}\right)\right.$ at the blue cross, which is close to the maximum of $\psi$ in Plot b) and lies within the magnetic resonance "island"). Plot d) shows the ratio $\psi(I, q, 4 \chi / \pi=1) / \psi(I, q, \chi=0)$. Circular polarization at $\theta=\pi / 2$ only improves the return flux at Paranal if $I \geq 10 \mathrm{~W} / \mathrm{m}^{2}$ (at smaller $\theta$, there is a much higher gain, as indicated by Fig. 3). The improvement at the blue cross is 1.52 (with the $B$-field at SOR, it would be only 1.2 , but can reach 2.0 for smaller $\theta$ ). We have limited the plot range in the lower right corner of d) to 1.8 for better rendering; in reality, the value exceeds 3. Plot e) shows the same as b), but for the $B$-field of SOR. As noted in Fig. 9, the optimal $q$ lies closer to $19 \%$ under this condition. 


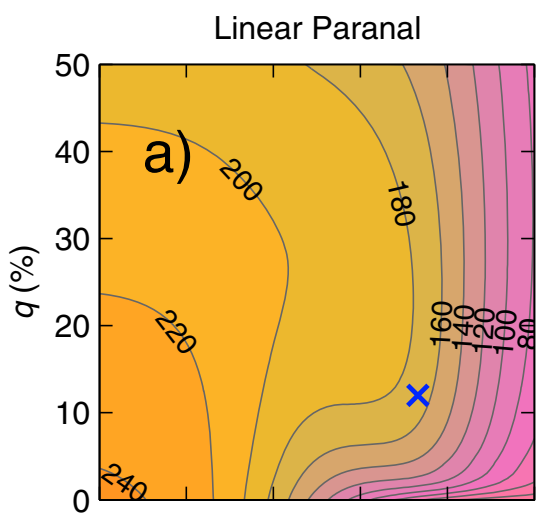

Circular/Linear Paranal

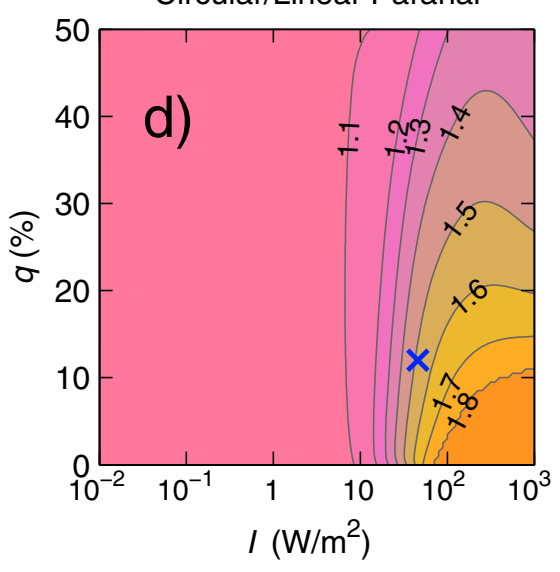

Circular Paranal

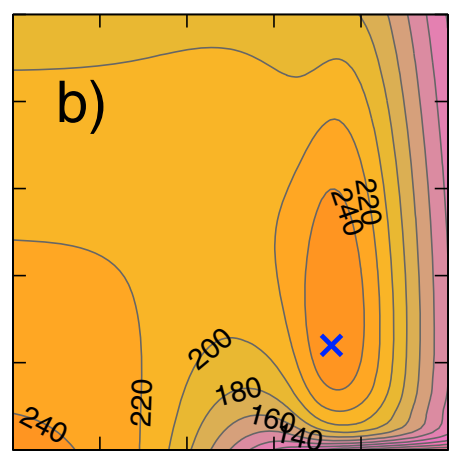

Circular SOR

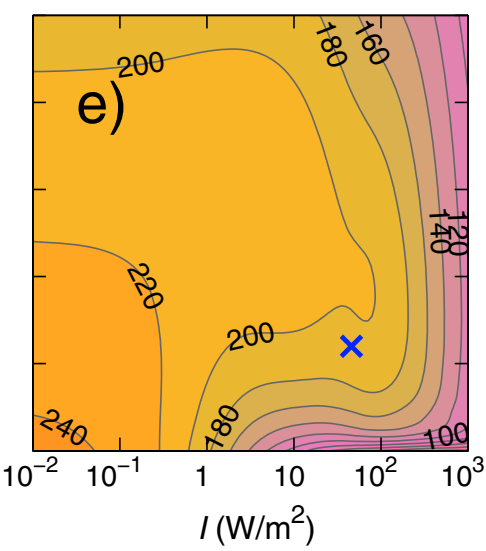

Repumping gain Paranal

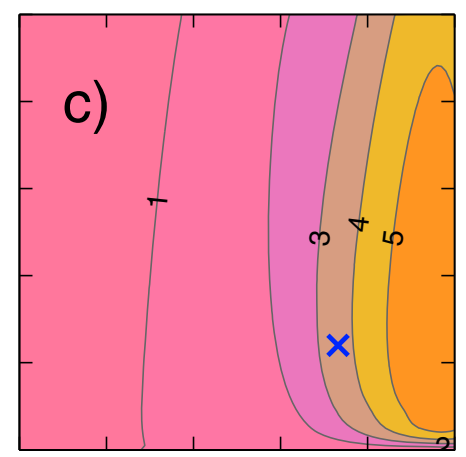

Repumping gain SOR

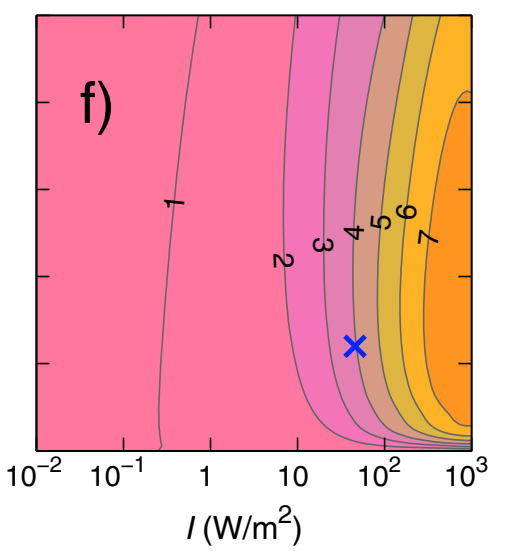

Fig. 10. a), b) Contour plots of $\psi(I, q)$ in $\mathrm{ph} / \mathrm{s} / \mathrm{sr} /\left(\mathrm{W} / \mathrm{m}^{2}\right)$ at Paranal for linear and circular polarization, respectively; d) the ratio $\psi($ circular $) / \psi$ (linear) at Paranal; e) same as b), but for SOR geomagnetic field; c),f) repumping gain $\psi(I, q) / \psi(I, q=0)$ at Paranal and SOR, respectively, circular polarization. Blue crosses: $I=46 \mathrm{~W} / \mathrm{m}^{2}, q=0.12$.

Note that even at $q=0.5$ (equal power in $\mathrm{D}_{2} \mathrm{a}$ and $\mathrm{D}_{2} \mathrm{~b}$ ), $\psi \approx 164 \mathrm{ph} / \mathrm{s} / \mathrm{sr} /\left(\mathrm{W} / \mathrm{m}^{2}\right)$ is not much below its optimum of 201 . Plot c) depicts the gain in $\psi$ when turning on repumping at standard conditions, i.e., $\psi(I, q) / \psi(I, q=0)$. Plot d) shows the same for SOR. At the blue crosses $\psi(q=0.12) / \psi(q=0)$ equals 3.6 and 4 , respectively.

\subsubsection{Laser linewidth}

So far, we have only simulated single-frequency line lasers. We now turn to lasers with Lorentzian lines of finite bandwidth. Figure 11 shows two contour plots of $\psi(I, \Delta f)$ for conditions at Paranal and SOR. The specific return is highest near the dashed blue lines, hence near $10 \mathrm{~W} / \mathrm{m}^{2} / \mathrm{MHz}\left(100 \mathrm{~W} / \mathrm{m}^{2}\right.$ per natural linewidth of sodium of $9.8 \mathrm{MHz}$ ) for Paranal and near $17 \mathrm{~W} / \mathrm{m}^{2} / \mathrm{MHz}$ for SOR. For irradiances below about $20 \mathrm{~W} / \mathrm{m}^{2}$, this linearity does not hold anymore and $\Delta f \rightarrow 0$ will yield higher return flux. Conversely, at $I \gg 1000 \mathrm{~W} / \mathrm{m}^{2}, \Delta f$ is no longer negligible compared to the Doppler width, and hence the absorption cross section falls off in the wings of the laser spectrum (our simulations for $q>0$ lose accuracy for wide bandwidths, see the discussion of Fig. 1). In the upper left region of the plot, $\psi$ decreases due to low pumping that is too weak to overcome the population equalization from Larmor precession, and in the lower right we suffer from saturation. We note that d'Orgeville (2000) has conducted a related study (d'Orgeville's Fig. 8 differs from ours in that its horizontal axis shows laser power and not irradiance, and it is rendered in double logarithmic scale).
Based on the dashed blue lines in Fig. 11, the optimum FWHM laser bandwidth can be estimated by $\Delta f_{\mathrm{opt}} \approx$ $\left(0.025 I_{P / 2}-0.5\right) / B$, where $\Delta f_{\text {opt }}$ is in $\mathrm{MHz}, I_{P / 2}$ in W/m $/ \mathrm{m}^{2}$ with $20 \leq I_{P / 2} \leq 1000 \mathrm{~W} / \mathrm{m}^{2}$, and $B$ in Gauss. We expect this optimal spectral power density to only depend on the relaxation time scales. This scaling relationship is good news for future LGS systems, since it means that we can achieve high return flux even at very large irradiances, as long as we keep increasing the laser bandwidth. Lasers with microsecond pulses designed for mesospheric spot tracking or lidar with duty cycles of 1:20-1:100, and hence high peak powers, may take advantage of this possibility.

\subsection{Optimization of laser beams}

In this subsection, we extend the analysis to entire laser beams by carrying out weighted integrals of $\psi(I)$ for (Gaussian) spot profiles, from which we compute $s_{\text {ce }}$ (Eq. (7)).

To begin, we conduct a comparison with the beam return flux computed by Milonni (1999). We apply our Bloch code to Milonni's Na conditions of $B=0.5 \mathrm{G}, q=0, \theta=30^{\circ}, \zeta=0^{\circ}$, $P=1 \mathrm{~W}, 2 \times I_{P / 2}=0.52 \mathrm{~W} / \mathrm{m}^{2}$, circular polarization $(4|\chi| / \pi=$ $1)$, no recoil, $\gamma_{\mathrm{ex}}=0, T_{\mathrm{Na}}=200 \mathrm{~K}, T_{\mathrm{a}}=1.0, \gamma_{\mathrm{vcc}}=0, \gamma_{S}=$ $1 /(100 \mu \mathrm{s}))$ and obtain $s_{\mathrm{ce}}=335 \mathrm{ph} / \mathrm{s} / \mathrm{W} /\left(\right.$ atoms $\left./ \mathrm{m}^{2}\right)$, in contrast to Milonni's value $s_{\mathrm{ce}}=240 \mathrm{ph} / \mathrm{s} / \mathrm{W} /\left(\right.$ atoms $\left./ \mathrm{m}^{2}\right)$ from Eq. (30) of that paper. If we switch to $\gamma_{S}=1 /(640 \mu \mathrm{s})$ (long-dashed curve in Milonni's Fig. 6a), we obtain $s_{\mathrm{ce}}=359 \mathrm{ph} / \mathrm{s} / \mathrm{W} /\left(\right.$ atoms $\left./ \mathrm{m}^{2}\right)$. For the combination $\gamma_{\mathrm{vcc}}=1 /(100 \mu \mathrm{s}), \gamma_{S}=1 /(640 \mu \mathrm{s})$, we find $s_{\mathrm{ce}}=303 \mathrm{ph} / \mathrm{s} / \mathrm{W} /\left(\right.$ atoms $\left./ \mathrm{m}^{2}\right)$, underlining the importance of properly modeling diffusion in atom velocity space. We suspect 

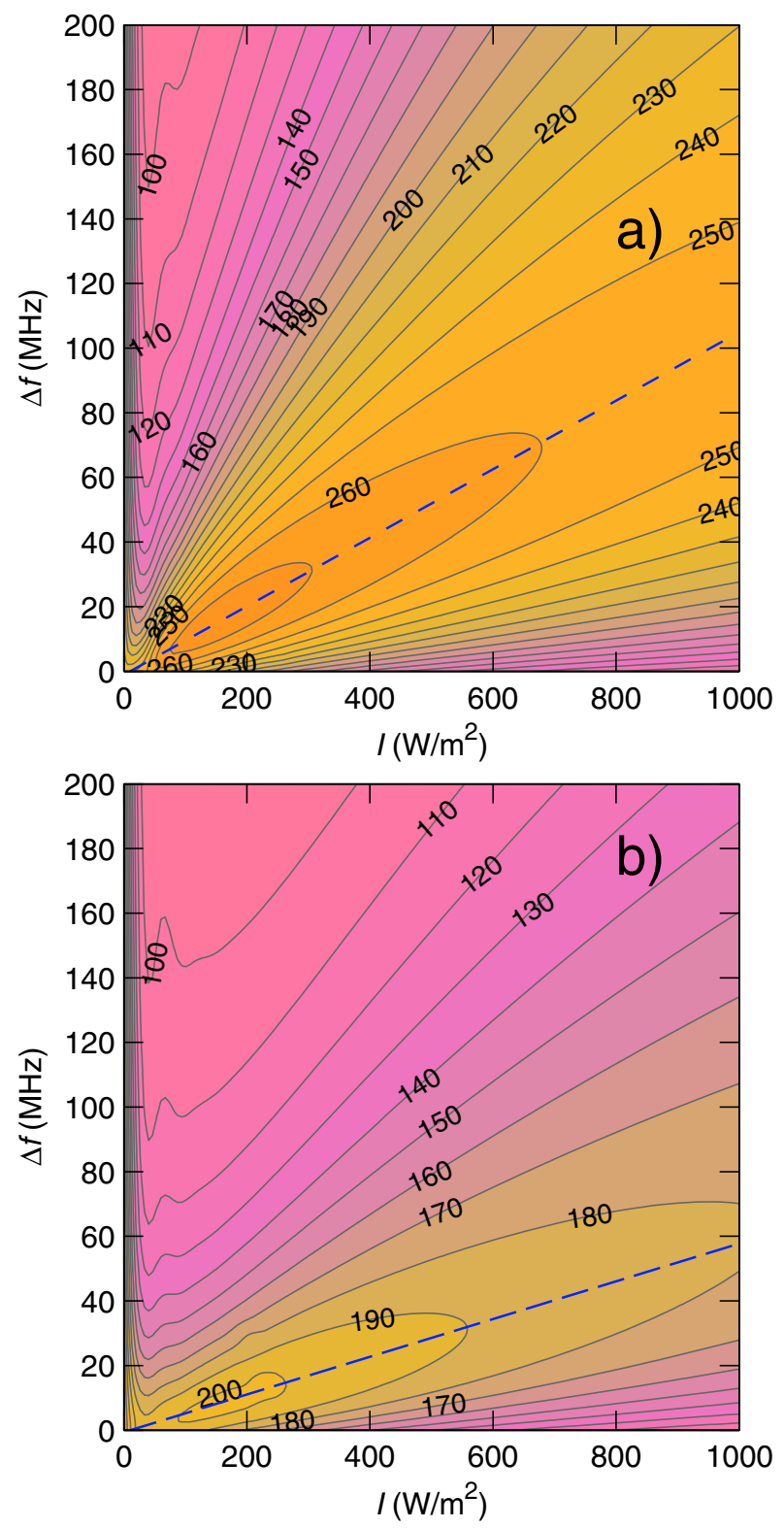

Fig. 11. a) Contour plot of $\psi(I, \Delta f)$ in $\mathrm{ph} / \mathrm{s} / \mathrm{sr} /\left(\mathrm{W} / \mathrm{m}^{2}\right)$ for standard conditions (Paranal). b) Same, but for the geomagnetic field of SOR. The optimal spectral irradiance is near 10 and $17 \mathrm{~W} / \mathrm{m}^{2} / \mathrm{MHz}$, respectively (dashed blue lines).

that the discrepancy in the flux results is partly due to Milonni's (1999) greatly simplified S-damping formula Eq. (18), which tends to overestimate spin relaxation.

Figure 12 shows a plot of the beam efficiency $s_{\text {ce }}$ for different geomagnetic field strengths at $\zeta=30^{\circ}$ (solid red: Paranal, dashed green: Mauna Kea, dash-dotted blue: SOR). The dotted magenta and solid black lines show $s_{\text {ce }}$ for Paranal at $P=217 \mathrm{~W}\left(I_{P / 2}=500 \mathrm{~W} / \mathrm{m}^{2}\right)$ and $P=435 \mathrm{~W}\left(I_{P / 2}=\right.$ $\left.1000 \mathrm{~W} / \mathrm{m}^{2}\right)$, respectively. At these irradiances, the $s_{\mathrm{ce}}(\Delta f)$ peaks at $38 \mathrm{MHz}$ and $75 \mathrm{MHz}$, respectively, without losing much of its magnitude compared to $P=20 \mathrm{~W}$. We expect that pulsed lasers with pulse durations much longer than $16 \mathrm{~ns}$ can enjoy this high specific return. Note that the numerical similarity of $s_{\mathrm{ce}} \leq 245 \mathrm{ph} / \mathrm{s} / \mathrm{W} /\left(\right.$ atoms $\left./ \mathrm{m}^{2}\right)$ with typical values of $\psi$ in $\mathrm{ph} / \mathrm{s} / \mathrm{sr} /\left(\mathrm{W} / \mathrm{m}^{2}\right)$ is a mere coincidence, and the two quantities should not be confused.

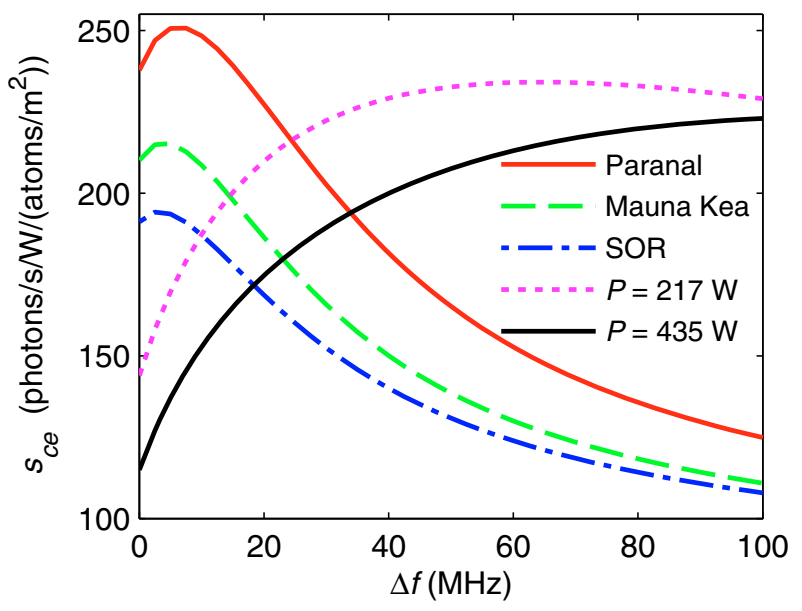

Fig. 12. Beam efficiency $s_{\text {ce }}$ as a function of $F W H M$ laser bandwidth $(\Delta f)$ for a $P=20 \mathrm{~W}$ laser at Paranal (solid red curve, standard conditions), Mauna Kea (dashed green), and SOR (dash-dotted blue), depending on the local geomagnetic-field strengths $(P=20 \mathrm{~W}, \theta=\pi / 2)$. Dotted magenta (solid black): Laser at Paranal for $P=217 \mathrm{~W}(P=$ $435 \mathrm{~W})$.

We add the caveat that the laser bandwidth is defined here as the short-term bandwidth, as opposed to the apparent bandwidth obtained from a long-term spectral measurement of a laser whose central frequency is slowly drifting, or being chirped.

\section{Conclusions}

In this article, we describe a modeling method to accurately determine the photon return flux from sodium LGS using Bloch (density matrix) equations. Numerical simulations are crucial, since it is not easy to achieve the desired optimization experimentally.

We summarize some optimization conclusions for $\mathrm{cw}$ sodium LGS lasers as follows:

1. The next generation of $20 \mathrm{~W}$-class (launched) $\mathrm{cw}$ laser sodium LGS will achieve unprecedented mesospheric irradiances near $I_{P / 2}=50 \mathrm{~W} / \mathrm{m}^{2}\left(I_{P / 2}=\right.$ "50\% power in the bucket irradiance") in good seeing conditions, using launch telescopes with $30-50 \mathrm{~cm}$ clear aperture diameter.

2. Atomic Larmor precession due to the geomagnetic field $B$ can completely suppress optical pumping and thus strongly reduce the LGS return flux, depending on the orientation between the laser beam and the field lines. LGS systems must be dimensioned for the beam pointing that yields the lowest return, i.e., laser beam orthogonal to $\boldsymbol{B}$.

3. In the future LGS irradiance regime, circular polarization will significantly elevate the return flux, with a rather relaxed polarization extinction ratio of PER $\leq 6.4 \mathrm{~dB}$. The polarization chirality (sense) does not matter.

4. Repumping (excitation of the $\mathrm{D}_{2} \mathrm{~b}$ line) with $10-20 \%$ of the laser power in combination with circular polarization increases the return flux further by a factor of 3-4. Repumping can moreover alleviate spatial power-broadening and hence reduce the observed LGS spot size.

5. The optimum $F W H M$ laser bandwidth can be estimated for a Lorentzian line shape by $\Delta f_{\text {opt }} \approx\left(0.025 I_{P / 2}-0.5\right) / B$, where $\Delta f_{\text {opt }}$ is in $\mathrm{MHz}, I_{P / 2}$ in $\mathrm{W} / \mathrm{m}^{2}\left(20 \leq I_{P / 2} \leq 1000 \mathrm{~W} / \mathrm{m}^{2}\right)$, and $B$ in Gauss. As a rule of thumb, the laser bandwidth in $\mathrm{MHz}$ should approximately equal the launched laser power in Watt divided by six, assuming a diffraction-limited spot 
size in good seeing. Employing lasers with a single line of $2-3 \mathrm{GHz}$ bandwidth is usually several times less efficient.

6. A laser with the above properties can achieve a specific return of $s_{\mathrm{ce}}=200-250$ photons $/ \mathrm{s} / \mathrm{W} /\left(\right.$ atoms $\left./ \mathrm{m}^{2}\right)$ at a low- $B$ location such as in northern Chile and $s_{\mathrm{ce}}=$ 150-200 photons $/ \mathrm{s} / \mathrm{W} /\left(\right.$ atoms $\left./ \mathrm{m}^{2}\right)$ at a high- $B$ location such as New Mexico (all values for laser beam orthogonal to $\boldsymbol{B}$ ).

We expect a numerical accuracy of $\pm 10 \%$ in our results of $\psi$ for a given parameter set. However, many physical simulation parameters such as spin relaxation and velocity-changing collision cross sections should be measured more accurately (at mesospheric temperatures). The corresponding relaxation rates depend on gas pressure and temperature and thus vary exponentially with altitude. Moreover, the assumption that velocitychanging collisions are memoryless (complete velocity reset after scattering) is very strong and may need to be refined. In practice, the absolute return flux uncertainty is completely dominated by sodium abundance and altitude distribution fluctuations (for instance, raising the altitude by $6 \mathrm{~km}$ causes a reduction of $\gamma_{\mathrm{vcc}}$ from $1 /(35 \mu \mathrm{s})$ to $1 /(100 \mu \mathrm{s})$, diminishing $s_{\mathrm{ce}}$ from 245 to 205 photons $/ \mathrm{s} / \mathrm{W} /\left(\right.$ atoms $\left.\left./ \mathrm{m}^{2}\right)\right)$. The accurate return flux computation of a realistic LGS system requires averaging along the beam, accounting for altitude-dependent temperature, gas concentrations, and laser irradiance. Despite these caveats, we do not expect the qualitative conclusions of this work to change when the parameter values are made more precise.

We also compare the Bloch simulation results with our Monte Carlo rate-equation simulation Exciter and find good agreement. However, such Monte Carlo rate-equation methods are based on numerous assumptions, and they always need to be validated against more rigorous methods such as a Bloch equation calculation. Moreover, our Bloch code is orders of magnitude faster than Exciter, with the runtime advantage quickly growing with the desired level of accuracy. Our Bloch simulation code can simulate any alkali and is publicly available on our websites ${ }^{8}$.

In this work, we treat only steady-state sodium excitation with $\mathrm{cw}$ lasers. Pulsed lasers, with pulse durations close to or shorter than the sodium lifetime of $16 \mathrm{~ns}$, will be dealt with in a forthcoming publication. However, we surmise that our present results are valid for microsecond laser pulses that are useful for mesospheric spot tracking and lidar. On-sky experiments to validate our results with a mobile $20 \mathrm{~W}$-class laser unit are being prepared at ESO at the moment, and are expected to yield results in 2010.

In conclusion, our simulations indicate that we have only begun to realize the full capability of sodium LGS, to be harvested by upcoming generations of laser-assisted AO, both with cw and pulsed lasers. The future of sodium LGS, so to speak, looks very bright.

Acknowledgements. We thank Craig Denman, Paul Hillman, Peter Hoffmann, Victor Kartoshkin, Ed Kibblewhite, Peter Milonni, Steve Morgan, Brian Patton, John Telle, Yan Feng, Nourredine Moussaoui, and Luke Taylor for their valuable input. We would like to give special credit to Will Happer, the father of sodium LGS, for his generous advice. This work was supported in part by the NGA NURI program.

\section{References}

Andrews, L. C., \& Phillips, R. L. 2005, Laser Beam Propagation through Random Media (SPIE Press, Bellingham), 2nd edn.

Bellanger, V., Courcelle, A., \& Petit, A. 2004, Comp. Phys. Comm., 162, 143 Bradley, L. C. 1992, J. Opt. Soc. Am. B, 9, 1931

Budker, D., Gawlik, W., Kimball, D. F., et al. 2002, Rev. Mod. Phys., 74, 1153

McClelland, J. J., \& Kelley, M. H. 1985, Phys. Rev. A, 31, 3704

Corney, A. 1977, Atomic and Laser Spectroscopy (Oxford: Oxford University Press)

Cussler, E. L. 1997, Diffusion. Mass Transfer in Fluid Systems 2nd edn. (Cambridge: Cambridge University Press)

Denman, C. A., Drummond, J. D., \& Eickhoff, M. L. 2006, Proc. SPIE, 6272, $62721 \mathrm{~L}$

Dmitriev, S. P., Dovator, N. A., \& Kartoshkin, V. A. 2008, Tech. Phys. Lett., 34, 693

Drob, D. P., et al. 2008, J. Geophys. Res., 113, A12304

Drummond, J., Denman, C., \& Hillman, P. 2007, ed. S. Ryan, 1215, E67

Fishbane, P. M., Gasiorowicz, S. G., \& Thornton, S. T. 2005, Physics for Scientists and Engineers (Prentice-Hall, Upper Saddle River)

Fried, D. L. 1966, J. Opt. Soc. Am., 56, 1372

Guillet de Chatellus, H., Pique, J.-P., \& Moldovan, I. C. 2008, J. Opt. Soc. Am. A, 25, 400

Happer, W. 1972, Rev. Mod. Phys., 44, 169

Happer, W., \& Van Wijngaarden, W. A. 1987, Hyperfine Interactions, 38, 435

Happer, W., MacDonald, G. J., Max, C. E., \& Dyson, F. J. 1994, J. Opt. Soc. Am. A, 11, 263

Hillman, P. D., Drummond, J. D., Denman, C. A., \& Fugate, R. Q. 2008, Proc. of the SPIE, 7015, 70150L

Holzlöhner, R., Bonaccini Calia, D., \& Hackenberg, W. 2008, Adaptive Optics Systems, Proc. of the SPIE, 7015, 701521

Holzlöhner, R., Bonaccini Calia, D., \& Hackenberg, W. 2009, ESO Technical Report VLT-TRE-ESO-11875-3940, Issue 1 (available upon request)

Hubin, N. 2009, Proc. of AO for ELT conference, abstract 118

Kartoshkin, V. A. 1998, Opt. and Spectr., 85, 177

Milonni, P. W., \& Thode, L. E. 1992, Appl. Opt., 31, 785

Milonni, P. W., Fugate, R. Q., \& Telle, J. M. 1998, J. Opt. Soc. Am. A, 15, 217

Milonni, P. W., Fearn, H., Telle, J. M., \& Fugate, R. Q. 1999, J. Opt. Soc. Am. A, 16,2555

Morris, J. R. 1994, J. Opt. Soc. Am. A, 11, 832

Moussaoui, N., Holzlöhner, R., Hackenberg, W., \& Bonaccini Calia, D. 2009, A\&A, 501, 793

Moussaoui, N., Clemesha, B., et al. 2010, A\&A, in press

Nagengast, W., Nass, A., Grosshauser, C., Rith, K., \& Schmidt, F. 1998, J. Appl. Phys., 83, 5626

d'Orgeville, C., Rigaut, F., \& Ellerbroek, B. L. 2000, Proc. SPIE, 4007, 131

Patat, F. 2004, The Messenger, ESO Publication, 118, 11

Pique, J.-P., Moldovan, I. C., \& Fesquet, V. 2006, J. Opt. Soc. Am. A, 23, 2817 Pfrommer, T., Hickson, P., \& She, C.-Y. 2009, Geophys. Res. Lett., 36, L15831 Ramsey, A. T., \& Anderson, L. W. 1964, Il Nuovo Cimento, 32, 1151

Ressler, N. W., Sands, R. H., \& Stark, T. E. 1969, Phys. Rev., 184, 102

Simonich, D. M., Clemesha, B. R., \& Kirchhoff, V. W. 1979, J. Geophys. Res., 84,1543

Taylor, L., Friedenauer, A., et al. 2009, Proc. CLEO 2009, postdeadline PDA.7 Telle, J., Drummond, J., et al. 2006, Proc. of the SPIE, 6215, 62150K-1

Telle, J., Drummond, J., Hillman, P., \& Denman, C. 2008, Proc. of the SPIE, 6878, 68780G-1

Thomas, S. J., Gavel, D., Adkins, S., \& Kibrick, B. 2008, Proc. SPIE, 70155L

Ungar, P. J., Weiss, D. S., Riis, E., \& Chu, S. 1989, J. Opt. Soc. Am. B, 6, 2058

van der Vorst, H. A. 1992, SIAM J. Sci. Comp., 13, 631

Wright, M. R. 2004, An Introduction to Chemical Kinetics (Wiley)

\footnotetext{
${ }_{8}$ ESO Laser Systems Department website http://www.eso. org/sci/facilities/develop/lgsf/; LGS extension to Atomic Density Matrix package, http://budker . berkeley.edu/ADM/ (requires Mathematica v. 6 or higher).
} 